TRANSACTIONS OF THE

AMERICAN MATHEMATICAL SOCIETY

Volume 352, Number 4, Pages 1453-1492

S 0002-9947(99)02468-X

Article electronically published on September 9, 1999

\title{
SHEARED ALGEBRA MAPS AND OPERATION BIALGEBRAS FOR MOD 2 HOMOLOGY AND COHOMOLOGY
}

\author{
DAVID J. PENGELLEY AND FRANK WILLIAMS
}

\begin{abstract}
The mod 2 Steenrod algebra $\mathcal{A}$ and Dyer-Lashof algebra $\mathcal{R}$ have both striking similarities and differences arising from their common origins in "lower-indexed" algebraic operations. These algebraic operations and their relations generate a bigraded bialgebra $\mathcal{K}$, whose module actions are equivalent to, but quite different from, those of $\mathcal{A}$ and $\mathcal{R}$. The exact relationships emerge as "sheared algebra bijections", which also illuminate the role of the cohomology of $\mathcal{K}$. As a bialgebra, $\mathcal{K}^{*}$ has a particularly attractive and potentially useful structure, providing a bridge between those of $\mathcal{A}^{*}$ and $\mathcal{R}^{*}$, and suggesting possible applications to the Miller spectral sequence and the $\mathcal{A}$ structure of Dickson algebras.
\end{abstract}

\section{INTRODUCTION}

Mod 2 "lower indexed" algebraic operations $D_{i}(i \geq 0)$ arising via $\mathbf{F}_{2}$-equivariance and the quadratic construction were used by Steenrod [Stee1, Stee2, Stee3] to create "upper operations" $S q^{i}(i \geq 0)$ in the cohomology of spaces, and his approach was extended by Araki and Kudo [AK] to create "upper operations" $Q^{i}$ $(i \geq 0)$ in the homology of iterated loop spaces. Adem [Ade] deduced relations amongst the $S q^{i}$, giving us the Steenrod algebra $\mathcal{A}$, and Dyer and Lashof [DL] indicated how this could be done for the $Q^{i}$, giving us the Dyer-Lashof algebra $\mathcal{R}$, although these relations were first explicitly given by May [May2. The relations amongst the underlying algebraic operations $D_{i}$ were first given, implicitly, by May in [May1, as part of his unified algebraic approach to $\mathcal{A}$ and $\mathcal{R}$, but neither the explicit relations nor the (bi)algebra generated by these operations was studied until relatively recently. The first mention of this bigraded bialgebra, which we shall call the Kudo-Araki-May algebra $\mathcal{K}$ in recognition of their contributions just cited, occurs in a note by Smirnov $\mathrm{Sm}$ in 1987, but more extensive study of it has not occurred until the mid-1990's, in independent work by Postnikov [Po], BissonJoyal [BJ], and the present authors. Perhaps one reason for this delay is that in $\mathcal{K}$ the gradings on products are not additive in the now familiar sense in topology, but rather skew-additive on the "topological degree" via a linear combination involving also the "length degree". We will use the term bigraded algebra in this more general sense. Note also that we call $\mathcal{K}$ a bialgebra, and not a Hopf algebra, since, like the Dyer-Lashof algebra, it is not connected and thus has no conjugation.

Received by the editors December 2, 1997.

1991 Mathematics Subject Classification. Primary 55S99; Secondary 16W30, 16W50, 55S10, $55 \mathrm{~S} 12,57 \mathrm{~T} 05$.

Key words and phrases. Steenrod algebra, Dyer-Lashof algebra, bialgebras, sheared algebra map, Kudo-Araki-May algebra, Nishida relations. 
Our point of view here, rather distinct from the historical development, will be to obtain the Steenrod and Dyer-Lashof algebras directly from the bialgebra $\mathcal{K}$ via the basic conversion formulas

$$
\begin{aligned}
S q^{i} x_{k} & =D_{k-i} x_{k}, \\
Q^{i} y_{k} & =D_{i-k} y_{k}
\end{aligned}
$$

on classes $x_{k}$ of degree $-k$ in cohomology (negatively graded) and $y_{k}$ of degree $k$ in homology. This process creates two new bialgebras completely different from $\mathcal{K}$, but with topological degree more simply linked to their actions on spaces. Each conversion involves a minor miracle from this point of view, namely a lack of entanglement between $k$ and the relations between the new operations under composition.

We intend to show that $\mathcal{K}$ and its dual $\mathcal{K}^{*}$ are tractable, and have some clear advantages over $\mathcal{A}, \mathcal{R}$, and their duals, while providing a unifying view via the notion of "sheared algebra homomorphism". Moreover, the duality between $\mathcal{K}$ and $\mathcal{K}^{*}$ has a number of very nice features. We believe that in certain circumstances, such as for the Miller spectral sequence [Mill] and the $\mathcal{A}$ structure of Dickson algebras, it may be more productive to consider the module actions available over $\mathcal{K}$ (or comodules over $\mathcal{K}^{*}$ ), rather than over $\mathcal{A}$ or $\mathcal{R}$. Our description is used to simplify the proofs of some technical propositions in the work of [GP] studying the $\mathcal{A}$-module structure of certain ideals in the algebras of Dickson invariants.

In section 2 below, we shall describe the generators and relations of $\mathcal{K}$ and list the principal results of this paper. In section 3 we shall see how $\mathcal{K}$ acts on graded modules and graded algebras in topology, including its relation to the suspension isomorphism in cohomology, and the homology suspension on iterated loop spaces. We also examine the bialgebra structures of $\mathcal{K}$ and $\mathcal{K}^{*}$ and how they interact. In section 4 we study actions of $\mathcal{K}$ on itself and on $\mathcal{K}^{*}$, arising from the Nishida relations, including a description which may shed light on the $\mathcal{A}$-module structure of the Dickson algebras, and we present a formula analogous to the Thom isomorphism theorem. In section 5 we formalize what we mean by a sheared algebra map, prove a general theorem for producing sheared algebra maps on Poincaré-Birkhoff-Witt algebras, and apply it to show the exact relationship between $\mathcal{K}$ and the Dyer-Lashof algebra, and between their duals. In section 6 we provide the precise relationship between $\mathcal{K}$ and the Steenrod algebra via another sheared algebra bijection with domain an algebra $\mathcal{K}(\infty)$ constructed from $\mathcal{K}$. In section 7 we use this to shed light on the tantalizing similarities and puzzling differences between the coproduct formulas in $\mathcal{K}^{*}$ and $\mathcal{A}^{*}$. In section 8 we compute the cohomology of the algebra $\mathcal{K}$ and show how it is closely related to $\mathcal{K}(\infty)$ and $\mathcal{A}$, again via sheared algebra bijections. We also examine the effect of sheared algebra maps in homology and cohomology, summarize how all our sheared algebra bijections are connected to each other, and mention possible applications via the Miller spectral sequence. Section 9 discusses future directions for research and applications. As an illustration, we show that a theorem of J. Lin about finite $H$-spaces can be reformulated elegantly in terms of $\mathcal{K}$.

We note that some previous work involves certain aspects of $\mathcal{K}$, for instance in providing useful descriptions of the homology of iterated loop spaces CCPS, CPS, CLM, MM], and calculating the mod $p$ homology of topological Hochschild homology $[\mathrm{H}]$. In $[\mathrm{Sm}], \mathcal{K}$ is characterized as the free radical Hopf algebra on a generator of dimension one. In $[\mathrm{PO}]$, Theorem 2 gives the product and coproduct in $\mathcal{K}^{*}$, cf. Theorems $\mathrm{B}$ and $\mathrm{C}$ below. And $[\mathrm{BJ}]$ includes the product, coproduct, and 
Nishida action in $\mathcal{K}$ as a main (and motivating) example in their study of $Q$-rings. Perhaps both $\mathcal{K}$ and the concomitant notion of sheared algebra homomorphism will find more widespread use.

We thank Dennis Sjerve for conversations with the second author which were the genesis of this project. We also thank Bob Bruner, Vince Giambalvo, Chuck McGibbon, Haynes Miller, Jack Morava, Peter May, Bill Singer, Jim Stasheff, and the referee for useful comments on earlier versions of this paper.

\section{Statements of PRINCIPAL RESUlts}

For each principal result we indicate in parentheses which section contains its proof.

Definition 2.1. We define our bigraded bialgebra $\mathcal{K}=\mathcal{K}_{*, *}$ to be the $\mathbf{F}_{2}$-algebra with identity in bidegree $(0,0)$ and generated by elements $D_{i} \in \mathcal{K}_{1, i}(i=0,1, \ldots)$, subject to the (Adem) relations

$$
D_{i} D_{j}=\sum_{k}\left(\begin{array}{c}
k-1-j \\
2 k-i-j
\end{array}\right) D_{i+2 j-2 k} D_{k}, \quad(i>j) .
$$

(Observe here that the binomial coefficient is zero unless $\frac{i+j}{2} \leq k<i$.)

The bidegree of elements in $\mathcal{K}$ is defined inductively by the requirement that the multiplication be a map

$$
\mathcal{K}_{m, i} \otimes \mathcal{K}_{n, j} \rightarrow \mathcal{K}_{m+n, i+2^{m}{ }_{j}}
$$

This definition is viable since the inductive formula is clearly consistent with the Adem relations and with associativity. We will call the first of the bidegrees the length, and the second the topological, degree. We shall frequently use the notation | | to denote topological degree.

Finally, the coproduct of $\mathcal{K}$ is defined on generators by the usual Cartan formula

$$
\phi\left(D_{i}\right)=\sum_{t=0}^{i} D_{t} \otimes D_{i-t},
$$

and we shall see that the product and coproduct maps of $\mathcal{K}$ interact as in a bialgebra. We note that $\mathcal{K}$ is a cocommutative component coalgebra [CLM, p. 18] with counit $D_{0}^{n}$ in the $n$-th component $\mathcal{K}_{n}=\mathcal{K}_{n, *}$. The behavior of the coproduct on bidegrees is

$$
\phi: \mathcal{K}_{n, i} \rightarrow \bigoplus_{t}\left(\mathcal{K}_{n, t} \otimes \mathcal{K}_{n, i-t}\right),
$$

which one sees by induction on $n$.

The 'skew' additivity of multiplication on the topological degree is a very interesting feature, which we hope the reader will become convinced is well worth becoming comfortable with (see the relation to topology after Definition 3.4 and the categorical consequences of $[\mathrm{Sm}]$ ). In fact, Haynes Miller pointed out to us that the skew additivity can be interpreted as a grading of $\mathcal{K}$ over the nonabelian monoid constructed as the semidirect product of $\mathbb{Z}^{+}$with $\mathbb{Z}^{+}$using the homomorphism $\varphi: \mathbb{Z}^{+} \rightarrow \operatorname{End}\left(\mathbb{Z}^{+}\right)$given by $\varphi(t)(x)=2^{t} x$.

As with $\mathcal{A}$ and $\mathcal{R}, \mathcal{K}$ also possesses a vector space basis of "admissible monomials" $D_{i_{1}} \cdots D_{i_{n}}$ where $i_{1} \leq \cdots \leq i_{n}$. We shall find it convenient to adopt the convention 
that $D_{i}=0$ for any $i$ that is not a non-negative integer. As mentioned in the introduction, $\mathcal{K}$, like $\mathcal{R}$, has no conjugation since it is not connected.

We next move to the structure of $\mathcal{K}^{*}$. The first step is

Lemma A (\$3). The elements $D_{0}^{i} D_{1}^{j}(i+j=n)$ form a basis for the coalgebra primitives of $\mathcal{K}_{n}$.

From the lemma we see that $\mathcal{K}^{*}$ is generated as an algebra by elements $x_{i, j}$ dual to the elements $D_{0}^{i} D_{1}^{j}$ with respect to the basis of admissible monomials. In fact, we prove

Theorem B (\$3). The algebra summand $\mathcal{K}_{n}^{*}$ is isomorphic to the polynomial algebra

$$
\mathbf{F}_{\mathbf{2}}\left[x_{i, j} \mid i+j=n, 0 \leq i \leq n-1\right]
$$

The identity element is $x_{n, 0} .\left(\right.$ Thus $\mathcal{K}_{n}^{*}$ is isomorphic to the Dickson algebra on $n$ generators [W].)

We also determine the coalgebra structure of $\mathcal{K}^{*}$ :

Theorem $\mathbf{C}(\$ 3)$. The coproduct in $\mathcal{K}^{*}$ is given by the formula

$$
\Delta\left(x_{i, j}\right)=\sum_{p, q} x_{i-p, j-q}^{2^{p}} \otimes x_{p, q} .
$$

The reader may wonder at the similarities and differences between this formula and the coproduct formula on Milnor's polynomial generators $\xi_{i}$ for $\mathcal{A}^{*}$ [Miln] Stee3. p. 133] or the formula of Madsen [Mad] for the coproduct in $\mathcal{R}^{*}$. These questions spurred much of our work, and are resolved in sections 5 and 7.

There are Nishida relations in $\mathcal{K}$, which give inductive formulas for a left (downward) action of the opposite algebra $\mathcal{K}^{\mathrm{op}}$ on $\mathcal{K}$,

$$
\mathcal{K}_{m, i}^{\mathrm{op}} \otimes \mathcal{K}_{n, j} \rightarrow \mathcal{K}_{n, \frac{i+j}{2^{m}}}
$$

We check that this Nishida action is a map of coalgebras, and thus the contragredient action

$$
\mathcal{K} \otimes \mathcal{K}_{n}^{*} \rightarrow \mathcal{K}_{n}^{*}
$$

makes $\mathcal{K}_{n}^{*}$ into an unstable algebra over the bialgebra $\mathcal{K}$.

We shall see that this action yields a formula analogous to the action of the Steenrod squares on the Thom class of vector bundles. Specifically, recall that if $S q$, the total Steenrod square, is defined by $S q=\sum_{i \geq 0} S q^{i}$, if $w$ is the total Stiefel-Whitney class of a vector bundle, and if $U$ is its Thom class, then there is the formula

$$
S q(U)=w \cdot U
$$

To state our analogy, let $x=\sum_{i, j \geq 0} x_{i, j}, \chi=\sum_{j \geq 0} x_{0, j}$, and $D=\sum_{i \geq 0} D_{i}$. Then we have

Theorem D. In the action of $\mathcal{K}$ on $\mathcal{K}^{*}$, there is the formula

$$
D(\chi)=x \cdot \chi
$$

This is a special case of the following theorem, which completely determines the action of $\mathcal{K}$ on $\mathcal{K}^{*}$, and thus encodes the action on the Dickson algebras (cf. $\mathrm{HP}, \mathrm{Mad}, \mathrm{W}]$ ). Since this description of the action is rather different from that of the Steenrod algebra on the dual Dyer-Lashof algebra, although equivalent to it, we hope it may be useful. 
Theorem E (\$4). For fixed $n$, and $0 \leq m \leq n$, let

$$
f_{m, n}^{+}=\sum_{k \geq m} x_{n-k, k}
$$

and

$$
f_{m, n}^{-}=\sum_{j<m} x_{n-j, j}
$$

in $\mathcal{K}_{n}^{*}$. Then

$$
D x_{n-m, m}=x_{n-m, m}^{2}+f_{m, n}^{+} f_{m, n}^{-} .
$$

Remark 2.2. While this theorem completely describes the action of $\mathcal{K}$ on $\mathcal{K}^{*}$, we note that the relation between this action and the coproduct in $\mathcal{K}^{*}$ remains mysterious.

One of the principal goals of this paper is to determine the interactions between the algebra structures of $\mathcal{K}, \mathcal{R}$, and $\mathcal{A}$, and to shed light on the similarities and differences between the formulas for the coproducts in their duals. To this end, we make the following definition.

Definition 2.3. We define a pre-algebra with shifting to be a quadruple $(K, \hat{K}, F, d)$ consisting of an algebra $K$, a vector space endomorphism $F$ (called the shift map), a specified set of algebra generators $\hat{K}$ (and thereby monomials in these generators), and a degree function $d$ from the set of elements which can be expressed as monomials to the nonnegative integers $\mathbb{Z}^{+}$.

Definition 2.4. Let $(K, \hat{K}, F, d)$ be a pre-algebra with shifting, $M$ be an algebra, and $\psi: K \rightarrow M$ be a linear transformation. We call $\psi$ a sheared algebra homomorphism if

$$
\psi\left(z z^{\prime}\right)=\psi\left(F^{d\left(z^{\prime}\right)}(z)\right) \psi\left(z^{\prime}\right)
$$

for any $z, z^{\prime} \in K$ with $z^{\prime}$ a monomial.

Remark 2.5. The shearing property may also be written as

$$
\psi \circ \mu_{K}=\mu_{M} \circ(\psi \otimes \psi) \circ \gamma
$$

where $\gamma\left(z \otimes z^{\prime}\right)=F^{d\left(z^{\prime}\right)}(z) \otimes z^{\prime}$ is the "shearing" map.

Of course, an ordinary algebra map is a degenerate example, with $F$ being the identity.

If the pre-algebra with shifting satisfies certain technical hypotheses, which we describe later, we shall call $(K, \hat{K}, F, d)$ an algebra with shifting. These hypotheses guarantee the existence of "admissible relations" and a vector space basis of "admissible monomials." Our main result on sheared algebra homomorphisms is

Theorem F (\$5). Suppose that $(K, \hat{K}, F, d)$ is an algebra with shifting, that $M$ is an algebra, and that we are given a function $\psi: \hat{K} \rightarrow M$, such that if $k^{\prime} k^{\prime \prime}=\sum l_{m}^{\prime} l_{m}^{\prime \prime}$ is an admissible relation, then

$$
\psi\left(F^{d\left(k^{\prime \prime}\right)}\left(k^{\prime}\right)\right) \psi\left(k^{\prime \prime}\right)=\sum \psi\left(F^{d\left(l_{m}^{\prime \prime}\right)}\left(l_{m}^{\prime}\right)\right) \psi\left(l_{m}^{\prime \prime}\right) .
$$


Let $\psi: K \rightarrow M$ be the linear transformation defined on admissible monomials by the formula

$$
\psi\left(l_{1} \cdots l_{n}\right)=\psi\left(F^{d\left(l_{2} \cdots l_{n}\right)}\left(l_{1}\right)\right) \cdots \psi\left(F^{d\left(l_{n}\right)}\left(l_{n-1}\right)\right) \psi\left(l_{n}\right) .
$$

Then $\psi$ is a sheared algebra homomorphism.

The import of the theorem is that if $\psi$ respects admissible relations in a sheared fashion, then the formula for the function $\psi$ on admissible monomials also holds on arbitrary monomials.

The first application of this theorem will be to determine the relationship between $\mathcal{R}$ and $\mathcal{K}$. We define the shift map $\delta: \mathcal{R} \rightarrow \mathcal{R}$ by

$$
\delta\left(Q^{i_{1}} \cdots Q^{i_{n-1}} Q^{i_{n}}\right)=Q^{i_{1}-2^{n-1}} \cdots Q^{i_{n-1}-2} Q^{i_{n}-1}
$$

on admissibles (by definition, $Q^{i}=0$ if $i<0$ ), and let $t$ denote topological degree. Applying our main theorem to the data $\left(\mathcal{R},\left\{Q_{i}\right\}, \delta, t\right)$, we have

Theorem G (from Proposition 5.1 and Theorem 5.17). The map $\theta_{0}: \mathcal{R} \rightarrow \mathcal{K}$, given on admissibles by

$$
\theta_{0}\left(Q^{i_{1}} \cdots Q^{i_{n-1}} Q^{i_{n}}\right)=D_{i_{1}-i_{2}-\cdots-i_{n}} \cdots D_{i_{n-1}-i_{n}} D_{i_{n}},
$$

is a sheared algebra bijection and coalgebra isomorphism. In particular, the defining formula for $\theta_{0}$ holds on arbitrary products, not just admissibles.

We also dualize Theorem $\mathrm{G}$ to shed light on the similarities and differences between the coproduct formulae in $\mathcal{K}^{*}$ and $\mathcal{R}^{*}$.

Relating $\mathcal{K}$ to $\mathcal{R}$ in this way was relatively easy, but it is more challenging to relate $\mathcal{K}$ to the Steenrod algebra $\mathcal{A}$ via a sheared algebra bijection. We proceed as follows.

First, recall [AK, CLM] that for each $m>0$, and $0 \leq k \leq \infty$, a vector space basis for the coalgebra primitives in $H_{*} \Omega^{k} S^{m+k}$ can be identified with the admissible monomials in $\mathcal{K}$ involving only $D_{l}$ for $0 \leq l<k$, via the action on the fundamental class. When $k$ is finite, we will denote by $\mathcal{K}(k)$ the primitives in $H_{*} \Omega^{k} S^{m+k}$ under this identification:

Definition 2.6. Define $\mathcal{K}(k)$ to be the vector subspace of $\mathcal{K}$ spanned by the admissible monomials of the form $D_{0}^{i_{0}} D_{1}^{i_{1}} \cdots D_{k-1}^{i_{k-1}}$.

While it appears at first that $\mathcal{K}(k)$ is merely a vector subspace, in fact $\mathcal{K}(k)$ is a sub-bialgebra of $\mathcal{K}$.

We may now define a new algebra $\mathcal{K}(\infty)$ by

$$
\mathcal{K}(\infty)=\lim _{k} \mathcal{K}(k)
$$

under algebra homomorphisms $\alpha: \mathcal{K}(k) \rightarrow \mathcal{K}(k-1)$ given on generators by $\alpha\left(D_{i}\right)=$ $D_{i-1}$. (Recall that since $D_{t}=0$ whenever $t$ is not a nonnegative integer, this formula gives $\alpha\left(D_{0}\right)=D_{-1}=0$.)

Theorem $\mathbf{H}$ (from Theorem 6.11). There is a sheared algebra bijection

$$
\psi_{\infty}: \mathcal{K}(\infty) \rightarrow \mathcal{A} .
$$

We move on to examine the dual map $\psi_{\infty}^{*}: \mathcal{A}^{*} \rightarrow \mathcal{K}(\infty)^{*}$. 
Proposition I (from Proposition [7.3). A basis for $\mathcal{K}(\infty)^{*}$ is given by "monomials"

$$
x_{0, n}^{-l} x_{1, n-1}^{l_{1}} \cdots x_{n-1,1}^{l_{n-1}} \in \mathcal{K}(\infty)_{n}^{*} \quad \text { for } l>\sum_{i=1}^{n-1} l_{i} .
$$

(Here $n$ is a grading induced from the original length grading in $\mathcal{K}$.)

The notation $x_{0, n}^{-l} x_{1, n-1}^{l_{1}} \cdots x_{n-1,1}^{l_{n-1}}$ is intended to suggest an algebra in which $x_{0, n}$ has been inverted. In fact, in [BPW] it is shown that $\mathcal{K}(\infty)^{*}$ is a subspace of such an algebra.

Finding a complete formula for $\psi_{\infty}^{*}$ appears to be quite complicated and is related to the work of Campbell, Peterson, and Selick [CPS. However, we are able to compute the "leading terms," in the following sense.

Theorem $\mathbf{J}$ (\$7). Let $l=\sum_{i=1}^{n} l_{i}$. If $l_{n} \geq 1$, then modulo $\bigoplus_{m>n} \mathcal{K}(\infty)_{m}^{*}$

$$
\psi_{\infty}^{*}\left(\xi_{1}^{l_{1}} \cdots \xi_{n}^{l_{n}}\right)=x_{0, n}^{-l} x_{1, n-1}^{l_{1}} \cdots x_{n-1,1}^{l_{n-1}} .
$$

We caution that while $\mathcal{K}(\infty)^{*}$ is endowed with a coproduct dual to the multiplication in $\mathcal{K}(\infty)$, we do not claim any algebra structure for $\mathcal{K}(\infty)^{*}$. We also do not know how to compute the coproducts of all elements of $\mathcal{K}(\infty)^{*}$, but we can compute certain ones which, combined with our leading term theorem, will shed light on the similarities and differences between the coproducts in $\mathcal{A}^{*}$ and $\mathcal{K}^{*}$.

The last group of results deals with cohomology. We use methods of [Pr] to compute the cohomology $H^{*}(\mathcal{K})=\operatorname{Ext}_{\mathcal{K}}\left(\mathbf{F}_{2}, \mathbf{F}_{2}\right)$ of $\mathcal{K}$, and then prove

Theorem K (from Theorems 8.3 and 8.5). There exist sheared algebra bijections

$$
\omega: \mathcal{K}(\infty) \rightarrow H^{*}(\mathcal{K})
$$

and

$$
\nu: H^{*}(\mathcal{K}) \rightarrow \mathcal{A}
$$

Sheared algebra morphisms induce maps on homology and cohomology.

Theorem $\mathbf{L}(\sqrt[8]{8})$. Let $(K, \hat{K}, F, d)$ be an algebra with shifting, $M$ an algebra, and $\psi: K \rightarrow M$ a sheared algebra homomorphism. Then the map $\bar{B}(\psi): \bar{B}(K) \rightarrow$ $\bar{B}(M)$ defined for the reduced bar constructions by

$$
\bar{B}(\psi)\left(\left[z_{1}|\cdots| z_{n-1} \mid z_{n}\right]\right)=\left[\psi\left(F^{d\left(z_{2} \cdots z_{n}\right)}\left(z_{1}\right)\right)|\cdots| \psi\left(F^{d\left(z_{n}\right)}\left(z_{n-1}\right)\right) \mid \psi\left(z_{n}\right)\right]
$$

is a chain map, which then produces induced maps on homology and cohomology.

Finally, we summarize many of our results in a single commutative diagram (Figure [1). Before discussing it, recall from [KL1, $\mathrm{Pr}$, Mill, Lo] that there is an algebraic version $\mathcal{R}(-\infty)$ of the Dyer-Lashof algebra which has $\mathcal{R}$ as a quotient, 


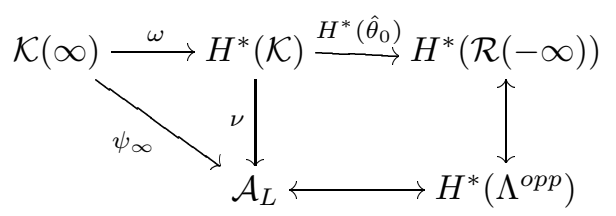

FiguRe 1.

is isomorphic to the opposite of the $\Lambda$-algebra, and whose cohomology algebra $H^{*}(\mathcal{R}(-\infty))$ is isomorphic to $\mathcal{A}_{L}$, the Lie Steenrod algebra, which is obtained by replacing $S q^{0}=1$ by $S q^{0}=0$ in the defining relations for $\mathcal{A}$.

All the maps in this diagram are degree-preserving isomorphisms of vector spaces, the labeled maps are sheared algebra bijections, and the unlabeled double-ended arrows are the algebra isomorphisms just mentioned. While $\omega$ and $\nu$ are both sheared algebra maps and $\psi_{\infty}=\nu \circ \omega$, it does not immediately follow that $\psi_{\infty}$ will be a sheared algebra map. In fact, $\psi_{\infty}$ and $\omega$ do not even use the same degree function on their common domain $\mathcal{K}(\infty)$. This phenomenon can be explained by a more detailed examination of the categorical structure of sheared algebra morphisms which we shall treat in a subsequent paper.

\section{Basic Properties of $\mathcal{K}$ and $\mathcal{K}^{*}$}

We begin this section with some instances of the relations in Definition 2.1.

Example 3.1. We have

$$
D_{2 i} D_{0}=D_{0} D_{i}, \quad D_{2 i+1} D_{0}=0, \quad D_{i} D_{i-1}=0 .
$$

We also can see, by checking binomial coefficients, that if the term $D_{0} D_{2^{a}}$ appears on the right hand side (RHS) of an Adem relation, then the left side must be $D_{2^{a+1}} D_{0}$, and that $D_{l} D_{l}$ does not appear on the RHS of any Adem relation. Finally, note that the RHS of the Adem relation for $D_{i} D_{j}$ involves only those $D_{l}$ with $l<i$.

We believe that the nice features of these sample relations already promise that the $\mathcal{K}$-Adem relations will sometimes be simpler to work with than those of $\mathcal{A}$ and $\mathcal{R}$.

To be certain that in our definition the product and coproduct interact as in a bialgebra, and that this is indeed the algebra of operations generated by the "algebraic Steenrod operations" $D_{i}$, we refer to May1 where these operations are defined in a setting which applies to both cohomology of spaces and homology of iterated loop spaces (see [CLM] for the latter). The symmetry that produces relations between iterated operations is expressed there by equation (e) in the proof of Theorem 4.7. The reader may find it satisfying to check that the somewhat elaborate equation there is merely a detailed expansion of the attractive formal power series symmetry identity

$$
D(u) D((u+v) v)=D(v) D((u+v) u),
$$

where $D(x)$ denotes the formal sum $\sum_{i \geq 0} D_{i} x^{i}$. We will call this identity the symmetric $\mathcal{K}$-Adem relations. Note that if we let $u, v$ have formal topological degree -1 , the skew-additivity of topological degree in $\mathcal{K}$ ensures that all the terms in the above relations are homogeneous of degree zero. The coproduct in $\mathcal{K}$ can be expressed in this context as

$$
\Delta D(x)=D(x) \otimes D(x) .
$$


Clearly this coproduct respects the symmetric $\mathcal{K}$-Adem relations, and thus does produce a bialgebra.

To extract the Adem relations of Definition 2.1 from the symmetric relations, we can use the clever residue method of [BM, Stei]. Letting $w=(u+v) v$ and noting that for constant $u, d w=u d v(\bmod 2$ of course $)$, we use the symmetry identity in the second equality of what follows:

$$
\begin{aligned}
D_{i} D_{j} & =\underset{u=0}{\operatorname{res}}\left[\underset{w=0}{\operatorname{res}} \frac{D(u) D(w)}{w^{j+1}} d w\right] \frac{d u}{u^{i+1}} \\
& =\underset{u=0}{\operatorname{res}}\left[\underset{v=0}{\operatorname{res}} \frac{D(v) D((u+v) u)}{u^{i}((u+v) v)^{j+1}} u d v\right] \frac{d u}{u} \\
& =\quad \underset{u=0}{\operatorname{res}}\left[\begin{array}{r}
\operatorname{res} \\
v=0
\end{array} \sum_{k, l} D_{l} D_{k} v^{l-j} u^{k-i+1}(u+v)^{k-j-1} \frac{d v}{v}\right] \frac{d u}{u} \\
& =\underset{u=0}{\operatorname{res}} \sum_{k, l}\left(\begin{array}{c}
k-j-1 \\
j-l
\end{array}\right) D_{l} D_{k} u^{(k-i+1)+(k-j-1)-(j-l)} \frac{d u}{u} \\
& =\sum_{k}\left(\begin{array}{c}
k-j-1 \\
2 k-i-j
\end{array}\right) D_{i+2 j-2 k} D_{k} .
\end{aligned}
$$

Thus the relations in Definition 2.1 follow.

We must note, however (as did [May1, BM, Stei] in analogous situations for the Steenrod and Dyer-Lashof operations), that these relations are valid not only for $i>j$, but for all $i, j \geq 0$ (with $\left(\begin{array}{l}c \\ d\end{array}\right)$ interpreted as $(-1)^{d}\left(\begin{array}{c}-c+d-1 \\ d\end{array}\right)$ when $c<0$ ). Is it not possible then, that the unstated formulas for $i \leq j$ could impose additional relations beyond the "standard" ones in our definition?

Neither Adem nor Steenrod in the case of the Steenrod algebra Ade, Stee3, nor May in the case of the Dyer-Lashof algebra May2, express concern about this issue when they deduce explicit relations. In each case this concern was presumably obviated by topological knowledge of the time; Adem knew [Ade, p. 231] from Serre's calculations of the cohomology of Eilenberg-Mac Lane spaces that the admissible monomials in Steenrod squares formed a basis for all operations, and May knew May2 similarly, from calculation of the homology of $Q S^{0}$, that the admissible monomials in Dyer-Lashof operations applied to the fundamental class are independent. Thus in each case no further relations were possible.

In our case we may also appeal to the homology of $Q S^{0}$, since the isomorphism $\theta_{0}$ of Theorem $\mathbb{G}$ (with $\mathcal{K}$ subject only to the "standard" relations of Definition 2.1) commutes with the actions of $\mathcal{R}$ and $\mathcal{K}$ on a zero-dimensional homology class. Thus the algebra of operations has no more relations than those in our definition. Having gone to all this trouble, it would nonetheless be very nice to have purely algebraic reasoning for why the relations for $i \leq j$ must be totally redundant, but at present we know of none.

It is also very useful to have these asymmetric $\mathcal{K}$-Adem relations expressed using formal power series, as follows. In the symmetric $\mathcal{K}$-Adem relations identity, let $w=(u+v) v$, and seek to eliminate $v$ from the identity. First, write the quadratic $v^{2}+u v+w=0(\bmod 2)$ as $\left(\frac{v}{u}\right)^{2}+\frac{v}{u}+\frac{w}{u^{2}}=0$. Then $v=u f\left(\frac{w}{u^{2}}\right)$ where $f(x)$ is any mod 2 formal power series solution to $f^{2}+f+x=0$. This leads to the asymmetric 
$\mathcal{K}$-Adem relations identity

$$
\begin{aligned}
D(u) D(w) & =D\left(u f\left(\frac{w}{u^{2}}\right)\right) D\left(u^{2}\left(1+f\left(\frac{w}{u^{2}}\right)\right)\right) \\
& =D\left(u f\left(\frac{w}{u^{2}}\right)\right) D\left(\frac{w}{f\left(\frac{w}{u^{2}}\right)}\right) .
\end{aligned}
$$

Working with this identity involves choosing a solution $f(x)$ for the quadratic, and being able to identify the coefficients in its integer powers as well. Of course, there are many integral combinatorial choices for the mod 2 solutions. This important quadratic is well known and studied, and Lemma 4.7 of GPR points to much of what one needs to know. For instance, one can obtain the $\mathcal{K}$-Adem relations of our definition by this approach, rather than by the Bullett-Macdonald residue method we used above.

The behavior of the cohomology of a space or the homology of an infinite loop space in relation to $\mathcal{K}$ motivates the following definitions.

Definition 3.2. A graded module $M_{*}$ over $\mathcal{K}$ is one satisfying the degree requirement

$$
\mathcal{K}_{m, i} \otimes M_{j} \rightarrow M_{i+2^{m} j} .
$$

Note that $\mathcal{K}$ with its topological degree is thus a graded module over $\mathcal{K}$.

Definition 3.3. A graded module $M$ over $\mathcal{K}$, as above, is called unstable provided that

$$
D_{i}: M_{-i} \rightarrow M_{-i}
$$

is the identity for $i \geq 1$, and that

$$
D_{i}\left(M_{k}\right)=0 \quad(i>-k) .
$$

Definition 3.4. If in addition to being an unstable graded module over $\mathcal{K}$, as per Definition 3.3 $M$ is also a graded algebra (with the usual additive grading convention) over $\mathcal{K}$ (with respect to the coproduct $\phi$ ), then $M$ is called an unstable algebra over $\mathcal{K}$ if it satisfies the condition

$$
D_{0}(y)=y^{2}
$$

for all $y \in M$.

Remark 3.5. In May1 and [CLM] it is implicit that the cohomology of a space (graded negatively) and the homology of an infinite loop space are functors to the category of unstable algebras over $\mathcal{K}$.

At this point, traditional treatments of homology and cohomology operations define "upper-indexed" Steenrod or Dyer-Lashof operations in terms of the generators of $\mathcal{K}$, then develop the algebraic properties of the respective algebras of operations. We, however, shall continue to focus on the bialgebra $\mathcal{K}$ and its dual.

Lemma 3.6. The elements $D_{i_{1}} \cdots D_{i_{n}}, i_{1} \leq \cdots \leq i_{n}$, form a vector space basis for $\mathcal{K}_{n, *}$ (which we will call the basis of admissible monomials).

Proof. This follows from the Adem relations in a standard way, using a lexicographic ordering of the set of all multi-indices $\left(i_{1}, \ldots, i_{n}\right)$.

We next prove Lemma $\mathrm{A}$ from section 2 . 
Proof of Lemma A . Clearly the elements $D_{0}^{i} D_{1}^{j}$ are primitive, since $D_{0}^{i}$ is grouplike (i.e. $\left.\phi\left(D_{0}^{i}\right)=D_{0}^{i} \otimes D_{0}^{i}\right)$ and $D_{1} D_{0}=0$. Since

$$
\phi\left(D_{0} D_{I}\right)=\left(D_{0} \otimes D_{0}\right) \phi\left(D_{I}\right),
$$

we need only examine admissible monomials $D_{I}$ in which the first subscript is at least 1 . So let $D_{I}=D_{i_{1}} \cdots D_{i_{n}}$ be admissible, with $i_{1} \geq 1$ and $i_{n} \geq 2$. Now

$$
\begin{aligned}
\phi D_{I}= & D_{1}^{n} \otimes\left(D_{i_{1}-1} \cdots D_{i_{n}-1}\right) \\
& + \text { terms involving other basis elements. }
\end{aligned}
$$

The lemma follows.

Note that $D_{0}^{i} D_{1}^{j}$ has bidegree $\left(i+j, 2^{i}\left(2^{j}-1\right)\right)$.

Observe that $\mathcal{K}_{*, *}$ is not of finite type in the second bidegree (the elements $D_{0}^{i}$ all have zero topological degree). With this in mind, we define $\mathcal{K}^{*}$ to be the bigraded dual to $\mathcal{K}$, i.e. $\mathcal{K}_{n,-q}^{*}=\left(\mathcal{K}_{n, q}\right)^{*}$. We will continue to refer to the dual bidegrees of $\mathcal{K}^{*}$ as "length" and "topological" degrees, as in $\mathcal{K}$. Since $\mathcal{K}$ is the direct sum of the cocommutative coalgebras $\mathcal{K}_{n}, \mathcal{K}^{*}$ is the direct sum of the commutative algebras $\mathcal{K}_{n}^{*}$. From Lemma @ we saw that $\mathcal{K}^{*}$ was generated as an algebra by elements $x_{i, j}$ dual to the elements $D_{0}^{i} D_{1}^{j}$ with respect to the basis of admissible monomials. Of course, the bidegree of $x_{i, j}$ is $\left(i+j,-2^{i}\left(2^{j}-1\right)\right)$.

We now move to the proof of Theorem $B$.

Proof of Theorem $B$. We note that the multiplication in $\mathcal{K}_{n}^{*}$ obeys the usual (nonskewed) degree convention (i.e. is additive in the second subscript; see the degree behavior of $\phi$ in Definition 2.1). Furthermore, note that on admissibles the correspondence

$$
D_{i_{1}} \cdots D_{i_{n}} \rightarrow x_{0, n}^{i_{1}} x_{1, n-1}^{i_{2}-i_{1}} \cdots x_{n-1,1}^{i_{n}-i_{n-1}}
$$

provides a bijection of graded vector spaces between $\mathcal{K}_{n}$ and

$$
\mathbf{F}_{2}\left[x_{i, j} \mid i+j=n, 0 \leq i \leq n-1\right] .
$$

Since $\mathcal{K}_{n}^{*}$ is generated by $\left\{x_{i, j} \mid i+j=n, 0 \leq i \leq n-1\right\}$, the theorem follows.

We observe that since $x_{i, j}$ has topological degree $-2^{i}\left(2^{j}-1\right)$, already the $\mathcal{K}_{n}^{*}$ realize the graded Dickson algebras [W], even before we consider their natural module structure over $\mathcal{A}$ or $\mathcal{K}$ arising from the Nishida relations.

We now aim for a coproduct formula on the algebra generators $x_{i, j} \in \mathcal{K}^{*}$. To that end, the next lemma gives another very useful property of the primitive elements $D_{0}^{i} D_{1}^{j}$.

Lemma 3.7. If $D_{I}$ and $D_{J}$ are admissible monomials such that $D_{I} D_{J}=D_{0}^{i} D_{1}^{j}$, then there exist non-negative integers $a$ and $b$ such that

$$
D_{I}=D_{0}^{i-a} D_{2^{a}}^{j-b}, \quad D_{J}=D_{0}^{a} D_{1}^{b} .
$$

Proof. This follows from Example 3.1.

The algebra $\mathcal{K}$ has several interesting and useful self-maps. The next definition describes one of these.

Definition 3.8. Let $V: \mathcal{K} \rightarrow \mathcal{K}$ be the Verschiebung, the bialgebra map dual to the Frobenius (squaring) map on $\mathcal{K}^{*}$.

Remark 3.9. Note that $V$ is an epimorphism since the squaring map is one-to-one. 
Proposition 3.10. On algebra generators, $V$ is given by $V\left(D_{i}\right)=D_{i / 2}$.

(Recall our convention that $D_{i}=0$ for any $i$ that is not a non-negative integer.)

Proof. Since the Frobenius map on $\mathcal{K}_{*, *}^{*}$ preserves the length degree and doubles topological degree, we must have $V\left(D_{i}\right)=D_{i / 2}$ since $V$ is onto.

Remark 3.11. This shows that "halving", $D_{i} \rightarrow D_{i / 2}$, extends to a valid algebra endomorphism on $\mathcal{K}$, which is not immediately obvious from the explicit Adem relations, although it follows easily from the symmetric power series form of the relations since $V(D(u))=D\left(u^{2}\right)$.

We shall henceforth, for admissible elements $y \in \mathcal{K}$, use the notation $y^{*}$ for the dual element to $y$ with respect to the basis of admissible monomials in $\mathcal{K}$.

Corollary 3.12. Let $D_{J}$ be admissible. We have the formula

$$
\left(D_{J}^{*}\right)^{2^{a}}=D_{2^{a} J}^{*} .
$$

Proof. By Proposition 3.10, we may compute for $D_{I}$ admissible,

$$
\left\langle\left(D_{J}^{*}\right)^{2^{a}}, D_{I}\right\rangle=\left\langle\left(V^{*}\right)^{a}\left(D_{J}^{*}\right), D_{I}\right\rangle=\left\langle D_{J}^{*}, V^{a}\left(D_{I}\right)\right\rangle=\left\langle D_{J}^{*}, D_{I / 2^{a}}\right\rangle,
$$

which is nonzero if and only if $D_{I / 2^{a}}=D_{J}$, or equivalently, $D_{I}=D_{2^{a} J}$.

We are now in a position to prove Theorem C.

Proof of Theorem $\left[\right.$ Let $D_{I}$ and $D_{J}$ be admissibles. We compute

$$
\left\langle\Delta\left(x_{i, j}\right), D_{I} \otimes D_{J}\right\rangle=\left\langle x_{i, j}, D_{I} D_{J}\right\rangle,
$$

which is nonzero if and only if $D_{I} D_{J}=D_{0}^{i} D_{1}^{j}$.

By Lemma 3.7, this means that $D_{I}=D_{0}^{i-p} D_{2^{p}}^{j-q}$, and $D_{J}=D_{0}^{p} D_{1}^{q}$. So by Corollary 3.12, we see that the terms of $\Delta\left(x_{i, j}\right)$ are as claimed.

It is now interesting to observe that since clearly each $D_{i}$ is indecomposable in $\mathcal{K}$, each $x_{0,1}^{i}$ is a coalgebra primitive, which at first sight seems perplexing in an $\mathbf{F}_{2}$-bialgebra, but is in fact consonant with the world of multiple components.

The $\mathcal{K}^{*}$ coproduct has a very nice representation if we first define the formal power series

$$
(\Delta x)(u, v, w)=\sum_{\substack{0 \leq p \leq i \\ 0 \leq q \leq j}} x_{i-p,, j-q}^{2^{p}} \otimes x_{p, q} u^{2^{p+q}-1} v^{2^{i}} w^{2^{i+j-q}-2^{p}},
$$

and note that it determines the coproduct since the exponents of $u, v, w$ determine $i, j, p, q$ uniquely. Letting

$$
x(v, w)=\sum_{0 \leq l \leq n} x_{n-l, l} v^{2^{n-l}} w^{2^{n}-1},
$$

we have

Corollary 3.13. The coproduct in $\mathcal{K}^{*}$ is completely encoded by the identity

$$
(\Delta x)(u, v, w)=(1 \otimes x)((x \otimes 1)(v, w), u) .
$$

Note also, that if we assign formal topological degrees $|u|=|w|=1$ and $|v|=-1$, then both $x(v, w)$ and $(\Delta x)(u, v, w)$ are homogeneous of degree -1 .

We end this section with some further particularly interesting features of $\mathcal{K}$ and $\mathcal{K}^{*}$, some of which we shall use below. 
Theorem 3.14. For $0<j_{1}<\cdots<j_{r} \leq n$, we have the formula

$$
x_{n-j_{1}, j_{1}} \cdots x_{n-j_{r}, j_{r}}=\left(D_{0}^{n-j_{r}} D_{1}^{j_{r}-j_{r-1}} \cdots D_{r-1}^{j_{2}-j_{1}} D_{r}^{j_{1}}\right)^{*} .
$$

We preface the proof of this theorem with two lemmas.

Lemma 3.15.

$$
\sum_{i=1}^{r} i 2^{i-1}=(r-1) 2^{r}+1
$$

Proof. Easy induction.

Lemma 3.16. Let $0<j_{1}<\cdots<j_{r} \leq n$. If $D_{I} \in \mathcal{K}_{n, *}$, where $I=\left(i_{1}, \ldots, i_{n}\right)$, and if $\left|D_{I}\right|=\left|D_{0}^{n-j_{r}} D_{1}^{j_{r}-j_{r-1}} \cdots D_{r-1}^{j_{2}-j_{1}} D_{r}^{j_{1}}\right|$, and if $i_{n} \leq r-1$, then $D_{I}$ is not admissible.

Proof. We note that

$$
\left|D_{I}\right| \geq\left|D_{0}^{n-r} D_{1} D_{2} \cdots D_{r}\right|=2^{n-r} \sum_{i=1}^{r} i 2^{i-1}=(r-1) 2^{n}+2^{n-r},
$$

by Lemma 3.15. But clearly, if $D_{I}$ were to be admissible, then $\left|D_{I}\right| \leq i_{n}\left(2^{n}-1\right) \leq$ $(r-1)\left(2^{n}-1\right)$.

Proof of Theorem 3.14. Let $D_{I}$ be admissible. Write $x=x_{n-j_{1}, j_{1}} \cdots x_{n-j_{r}, j_{r}}, x^{\prime}=$ $x_{n-j_{1}, j_{1}-1} \cdots x_{n-j_{r}, j_{r}-1}$, and $I=\left(I^{\prime}, i_{n}\right)$.

From the coproduct formula for $\mathcal{K}^{*}$ it is immediate that $\left\langle x, D_{I}\right\rangle$ is zero if $i_{n}>r$. Furthermore, if $i_{n}=r$, we have

$$
\left\langle x, D_{I}\right\rangle=\left\langle\Delta(x), D_{I^{\prime}} \otimes D_{r}\right\rangle=\left\langle x^{\prime} \otimes x_{0,1}^{r}, D_{I^{\prime}} \otimes D_{r}\right\rangle=\left\langle x^{\prime}, D_{I^{\prime}}\right\rangle .
$$

If $j_{1}-1>0$, then we may conclude inductively (on $n$ ) that this is nonzero if and only if $D_{I^{\prime}}=D_{0}^{(n-1)-\left(j_{r}-1\right)} \cdots D_{r}^{j_{1}-1}$, whence $D_{I}=D_{0}^{n-j_{r}} \cdots D_{r}^{j_{1}}$.

If $j_{1}-1=0$, i.e. $j_{1}=1$, then $\left\langle x^{\prime}, D_{I^{\prime}}\right\rangle=\left\langle x_{n-j_{2}, j_{2}-1} \cdots x_{n-j_{r}, j_{r}-1}, D_{I^{\prime}}\right\rangle$, which, again inductively, is nonzero if and only if $D_{I^{\prime}}=D_{0}^{n-j_{r}} \cdots D_{r-1}^{j_{2}}$, whence again $D_{I}=D_{0}^{n-j_{r}} \cdots D_{r}^{j_{1}}$. So if $i_{n}=r$, the only possible $D_{I}$ is the desired one.

Finally, Lemma 3.16 applies to show that there are no admissibles $D_{I}$ in this bidegree with $i_{n} \leq r-1$. Hence if $D_{I}$ is admissible, then $\left\langle x, D_{I}\right\rangle$ is nonzero if and only if $D_{I}$ is as desired.

For more complex monomials in $\mathcal{K}^{*}$, the duality with admissibles in $\mathcal{K}$ becomes more elaborate. An algorithm for dealing with this question is given in [CPS.

Let $A$ be a bialgebra. An element $g \in A$ is called grouplike (cf. proof of Lemma (A) if

$$
\Delta(g)=g \otimes g
$$

where $\Delta$ denotes the coproduct in $A$.

Remark 3.17. If $g \in A$ is a grouplike element and if $f: A \rightarrow A$ is given by $f(a)=g a$, then $f$ is a morphism of coalgebras and (hence) $f^{*}: A^{*} \rightarrow A^{*}$ is a morphism of algebras.

Some useful self-maps of $\mathcal{K}$ and $\mathcal{K}^{*}$ are as follows: 
Example 3.18. In $\mathcal{K}$, the coproduct formula shows that the element $D_{0}$ and the formal sum $D=\sum_{i \geq 0} D_{i}$ are grouplike. We will denote the algebra maps dual to left multiplication by $D_{0}$ and $D$ by $\kappa^{*}$ and $\lambda^{*}$, respectively. From Lemma 3.7 their values on the algebra generators of $\mathcal{K}^{*}$ are

$$
\kappa^{*}\left(x_{i, j}\right)=x_{i-1, j}
$$

and

$$
\lambda^{*}\left(x_{i, j}\right)=x_{i-1, j}+x_{i, j-1} .
$$

In $\mathcal{K}^{*}$, the sum $\chi=\sum_{j \geq 0} x_{0, j}$ satisfies $\Delta(\chi)=\chi \otimes \chi$, so $\alpha: \mathcal{K} \rightarrow \mathcal{K}$ dual to multiplication by $\chi$ is an algebra map. As earlier with $V^{*}$, multiplication by $\chi$ preserves the length degree. Then the epimorphism $\alpha: \mathcal{K}_{1, i} \rightarrow \mathcal{K}_{1, i-1}$ must be given by

$$
\alpha\left(D_{i}\right)=D_{i-1}
$$

It is interesting to use the Adem relations to provide an alternative proof that this formula for $\alpha$ yields an algebra endomorphism. In fact, this follows trivially from their symmetric power series form; since

$$
\alpha(D(x))=x D(x)
$$

applying $\alpha$ to the symmetric identity simply multiplies both sides by $u(u+v) v$.

Remark 3.19. The map $\alpha$ has topological interpretations. If we regard $\mathcal{K}$ as operating on the cohomology of spaces and let $\Sigma: H^{*}(X) \rightarrow H^{*}(\Sigma X)$ be the suspension isomorphism, $x \in H^{*}(X)$, and $D_{I}$ be a monomial in $\mathcal{K}$, then since $\Sigma$ is essentially a cup product with a one-dimensional class, we check as in [Stee3, May1, that

$$
D_{I}(\Sigma(x))=\Sigma\left(\alpha\left(D_{I}\right)(x)\right) .
$$

Also, if $X$ is an infinite loopspace with $\mathcal{K}$ acting on it, $z \in H_{*}(\Omega X), \sigma_{*}$ is the homology suspension, and $D_{I}$ is a monomial in $\mathcal{K}$, then it follows from [AK, DL, CLM] that

$$
\alpha\left(D_{I}\right)\left(\sigma_{*}(z)\right)=\sigma_{*}\left(D_{I}(z)\right) .
$$

The self-maps we have described will be quite useful in describing the relation between $\mathcal{K}$ and the Dyer-Lashof and Steenrod algebras, as will the following proposition, which illustrates their use.

Proposition 3.20. If $D_{I} \in \mathcal{K}_{n, *}$ is admissible, then

$$
x_{0, n}^{a} D_{I}^{*}=D_{I+(a, \ldots, a)}^{*} .
$$

Proof. We compute, for admissible $D_{J}$,

$$
\left\langle x_{0, n}^{a} D_{I}^{*}, D_{J}\right\rangle=\left\langle\chi^{a}\left(D_{I}^{*}\right), D_{J}\right\rangle=\left\langle D_{I}^{*}, \alpha^{a}\left(D_{J}\right)\right\rangle,
$$

which is non-zero if and only if $\alpha^{a}\left(D_{J}\right)=D_{I}$, i.e. when $D_{J}=D_{I+(a, \ldots, a)}$. 


\section{NiSHIDA ACTIONS}

In $\mathcal{K}$, the Nishida relations [N, May2 May1, p. 209] [Mad, pp. 244-245] will give inductive formulas for a left (downward) action of the opposite algebra $\mathcal{K}^{\text {op }}$ on $\mathcal{K}$,

$$
\mathcal{K}_{m, i}^{\mathrm{op}} \otimes \mathcal{K}_{n, j} \rightarrow \mathcal{K}_{n, \frac{i+j}{2 m}}
$$

which we shall denote by $D_{I} * D_{J}$, to distinguish from multiplication in $\mathcal{K}$. Traditionally, the Nishida relations mediate the interaction of the $\mathcal{A}^{\text {op }}$ and $\mathcal{R}$ actions on the homology of an infinite loop space. If $X$ is an infinite loopspace and $x \in H_{*}(X)$, then in terms of $\mathcal{K}$ they take the form

$$
D_{s} *\left(D_{t} x\right)=\sum_{k}\left(\begin{array}{c}
\frac{t+s}{2} \\
\frac{t-s}{2}+k
\end{array}\right) D_{\frac{t+s}{2}-k}\left(D_{k} * x\right) .
$$

Here $D_{s} * y$ is the left downward action of $\mathcal{K}^{\mathrm{op}}$ on $H_{*}(X)$ that is equivalent to the standard left downward action of $\mathcal{A}^{\mathrm{op}}$ on $H_{*}(X)$. To obtain an action of $\mathcal{K}^{\mathrm{op}}$ on $\mathcal{K}$, we can use the action on a zero-dimensional homology class and define inductive formulas

$$
D_{s} * 1= \begin{cases}1, & \text { if } s=0 \\ 0, & \text { otherwise }\end{cases}
$$

and

$$
D_{s} *\left(D_{t} D_{J}\right)=\sum_{k}\left(\begin{array}{c}
\frac{t+s}{2} \\
\frac{t-s}{2}+k
\end{array}\right) D_{\frac{t+s}{2}-k}\left(D_{k} * D_{J}\right) .
$$

The reader may again be pleased to see that this formula is merely the formal power series identity

$$
D(u) *\left(D(v) \cdot \_\right)=D((u+v) v) \cdot\left(D((u+v) u) * \_\right)
$$

in different guise, where $D(x) *$ denotes $\sum D_{i} x^{i} *$. A straightforward computation, which we leave to the reader, shows that this formal power series formula for the action is compatible with (the opposite of) the formal power series symmetric $\mathcal{K}$ Adem relations in section 3 This provides satisfying confirmation that we have really described a bona fide algebra action of $\mathcal{K}^{\mathrm{op}}$ on $\mathcal{K}$, underlying the traditional Nishida relations for $\mathcal{A}^{\mathrm{op}}$ and $\mathcal{R}$. It is also interesting to compare our formulas with (3a) of [Stei].

As in the formal power series identity for the coproduct in $\mathcal{K}^{*}$, where both sides were homogeneous of degree -1 , in this case we find that both sides of our action formula have homogeneous degree zero if we let $|u|=|v|=-1 / 2$, where the degree of $D_{s} *\left(D_{t} \cdot{ }_{-}\right)$is $(t+s) / 2$, and that of $D_{l} \cdot\left(D_{k} *{ }_{-}\right)$is $l+k$, since these are the amounts by which they increase the degrees they are applied to.

We also wish to note at this point that by using the action based on fundamental classes in positive degrees, we obtain different actions of $\mathcal{K}^{\mathrm{op}}$ on $\mathcal{K}$. We shall consider these actions and their topological significance in a subsequent paper.

Example 4.1. Taking $t=0$, we obtain

$$
D_{s} *\left(D_{0} D_{J}\right)= \begin{cases}D_{0}\left(D_{s / 2} * D_{J}\right) & \text { if } s \text { is even; } \\ 0 & \text { if } s \text { is odd }\end{cases}
$$


Also,

$$
D_{s} *\left(D_{1} D_{J}\right)= \begin{cases}D_{1}\left(D_{\frac{1+s}{2}-1} * D_{J}\right) & \text { if } \frac{1+s}{2} \text { is even } \\ D_{0}\left(D_{\frac{1+s}{2}} * D_{J}\right)+D_{1}\left(D_{\frac{1+s}{2}-1} * D_{J}\right) & \text { if } \frac{1+s}{2} \text { is odd } \\ 0 & \text { if } s \text { is even }\end{cases}
$$

Further,

$$
D_{s} *\left(D_{t} D_{J}\right)=0 \quad \text { if } s, t \text { have different parity. }
$$

And finally,

$$
D_{0} * D_{J}=D_{J / 2},
$$

so we see that the action of $D_{0}$ produces the Verschiebung map $V$ of Definition 3.8

We next wish to observe, as pointed out in section 2 that dualizing from the Nishida action turns each $\mathcal{K}_{n}^{*}$ into an unstable algebra over $\mathcal{K}$ à la Definition 3.4 First note that the Nishida action is a map of coalgebras, as may be verified straightforwardly by induction on length in $\mathcal{K}$ using the formal power series formulation above of the Nishida action. Thus the contragredient action

$$
\mathcal{K} \otimes \mathcal{K}_{n}^{*} \rightarrow \mathcal{K}_{n}^{*}
$$

makes $\mathcal{K}_{n}^{*}$ into an algebra over the bialgebra $\mathcal{K}$. Moreover, we see by induction on length from the Nishida relations that $*$ satisfies (the dual of) the conditions in Definition 3.3. Finally, using Proposition 3.10 to dualize the last equation of Example 4.1 we obtain the squaring condition of Definition 3.4.

We shall prepare for the proof of Theorem $\mathbb{E}$ with a lemma illustrating computations using the Nishida relations of Example 4.1.

Lemma 4.2. Let $0 \leq a<d \leq a+b$ and $c \geq 0$. Then

$$
D_{2^{a+b}+2^{a}-2^{d}} *\left(D_{0}^{a} D_{1}^{b} D_{2}^{c}\right)=D_{0}^{d-1} D_{1}^{a+b+c-d+1} .
$$

Proof. By repeated applications of the formulas in Example 4.1, we compute

$$
\begin{aligned}
D_{2^{a+b}} & +2^{a}-2^{d} *\left(D_{0}^{a} D_{1}^{b} D_{2}^{c}\right) \\
& =D_{0}^{a}\left(D_{2^{b}+1-2^{d-a}} * D_{1}^{b} D_{2}^{c}\right) \\
& =D_{0}^{a}\left(D_{2^{d-a}\left(2^{b-d+a}-1\right)+1} * D_{1}^{b} D_{2}^{c}\right) \\
& =D_{0}^{d-1}\left(D_{2\left(2^{b-d+a}-1\right)+1} * D_{1}^{b-d+a+1} D_{2}^{c}\right) \\
& =D_{0}^{d-1} D_{1}\left(D_{2^{b-d+a}-1} * D_{1}^{b-d+a} D_{2}^{c}\right) \\
& =D_{0}^{d-1} D_{1}^{1+b-d+a}\left(D_{0} * D_{2}^{c}\right) \\
& =D_{0}^{d-1} D_{1}^{1+b-d+a+c} .
\end{aligned}
$$

Proof of Theorem $\underline{E}$. Since $\mathcal{K}$ acts unstably on $\mathcal{K}_{n}^{*}$, and $x_{n-m, m}$ lies in topological degree $-2^{n-m}\left(2^{m}-1\right)$ in $\mathcal{K}_{n}^{*}$, the element $D_{i} x_{n-m, m}$ can be nonzero only if it lies in a degree from $-2^{n-m+1}\left(2^{m}-1\right)$ through $-2^{n-m}\left(2^{m}-1\right)$. In degrees from $-\left(2^{n+1}-2\right)$ to 0 , a basis for $\mathcal{K}_{n}^{*}$ consists of the elements $x_{n-j, j} x_{n-k, k}$ for $0 \leq j \leq$ $k \leq n$, lying in the distinct degrees $2^{n-j}+2^{n-k}-2^{n+1}$, together with the elements $x_{n-1,1}^{2} x_{n-k, k}$ for $1 \leq k \leq n-1$, the latter lying respectively in the same degrees as $x_{n-k-1, k+1}^{2}$ in the first list.

Thus $D_{i} x_{n-m, m}$ lands in a degree of zero rank unless $i=2^{n-j}+2^{n-k}-2^{n-m+1}$ for $0 \leq j \leq k \leq n$, in which degree the dual is spanned by $D_{0}^{n-k} D_{1}^{k-j} D_{2}^{j}$, together 
with $D_{0}^{n-j+1} D_{1}^{j-2} D_{3}$ if $j=k \geq 2$ also. We complete the proof by considering four cases discriminating primarily where $m$ lies in relation to $j$ and $k$.

Case 1. If $j<m \leq k$, then in the degree of $D_{i} x_{n-m, m}, \mathcal{K}_{n}^{*}$ has rank one, and we compute

$$
\begin{aligned}
& \left\langle D_{2^{n-j}+2^{n-k}-2^{n-m+1}}\left(x_{n-m, m}\right), D_{0}^{n-k} D_{1}^{k-j} D_{2}^{j}\right\rangle \\
& \quad=\left\langle x_{n-m, m}, D_{\left.2^{n-j}+2^{n-k}-2^{n-m+1} * D_{0}^{n-k} D_{1}^{k-j} D_{2}^{j}\right\rangle}\right\rangle \\
& \quad=\left\langle x_{n-m, m}, D_{0}^{n-m} D_{1}^{m}\right\rangle=1,
\end{aligned}
$$

taking $a=n-k, b=k-j, c=j$, and $d=n-m+1$ in Lemma 4.2. Thus $D_{i} x_{n-m, m}=x_{n-j, j} x_{n-k, k}$ as claimed.

Case 2. If $j=m=k$, then $D_{0} x_{n-m, m}=x_{n-m, m}^{2}$, since $\mathcal{K}$ acts unstably on $\mathcal{K}^{*}$, producing the leading term in the formula claimed.

Case 3. If $j \leq k<m$, then for $j \geq 1, D_{i} x_{n-m, m}=0$ as claimed, since computing as in Case 1 using the formulas from Example 4.1, we obtain

$$
\begin{aligned}
& \left\langle D_{2^{n-j}+2^{n-k}-2^{n-m+1}}\left(x_{n-m, m}\right), D_{0}^{n-k} D_{1}^{k-j} D_{2}^{j}\right\rangle \\
& \quad=\left\langle x_{n-m, m}, D_{2^{n-j}+2^{n-k}-2^{n-m+1}} * D_{0}^{n-k} D_{1}^{k-j} D_{2}^{j}\right\rangle \\
& \quad=\left\langle x_{n-m, m}, 0\right\rangle \\
& \quad=0
\end{aligned}
$$

and, if also $j=k \geq 2$, then similarly

$$
\left\langle D_{2^{n-j+1}-2^{n-m+1}}\left(x_{n-m, m}\right), D_{0}^{n-j+1} D_{1}^{j-2} D_{3}\right\rangle=0 .
$$

For $j=0$ we see that the result follows from instability.

Case 4. If $m \leq j<k$ or $m<j \leq k$, then $i<0$, which cannot occur.

The action of $\mathcal{K}$ on $\mathcal{K}^{*}$, as described by Theorem E lends itself to formulation with formal power series. For $m \geq 0$, let

$$
\begin{aligned}
f_{m}^{+}(u, v, w) & =\sum_{m \leq k \leq n} x_{n-k, k} u^{2^{n-k}-2^{n-m}} v^{2^{n-m}} w^{2^{n}-1}, \\
f_{m}^{-}(u, v, w) & =\sum_{0 \leq j<m \leq n} x_{n-j, j} u^{2^{n-j}-2^{n-m}} v^{2^{n-m}} w^{2^{n}-1},
\end{aligned}
$$

and recall from section 3 the series

$$
x(v, w)=\sum_{0 \leq l \leq n} x_{n-l, l} v^{2^{n-l}} w^{2^{n}-1} .
$$

Notice that $x(v, w)=f_{m}^{+}(v, v, w)+f_{m}^{-}(v, v, w)$, a series independent of $m$. We have

Corollary 4.3. The action of $\mathcal{K}$ on $\mathcal{K}^{*}$ is completely encoded in the identity

$$
D(u) x\left(v^{2}, w^{2}\right)=[x(v, w)]^{2}+\sum_{m \geq 0} f_{m}^{+}(u, v, w) f_{m}^{-}(u, v, w) .
$$

With $|v|=-1=|u|$ and $|w|=1$, this expression is homogeneous of degree -2 . 


\section{Sheared algebra maps and the Relation Between $\mathcal{R}, \mathcal{K}$, AND THEIR DUALS}

In this section our focus will be on how $\mathcal{R}$ and $\mathcal{K}$ are related, with this relationship as a motivating example of a more general phenomenon, that of a "sheared algebra map", to be exhibited by several varied and important maps in this paper. Thus we will begin by examining the detailed relationship between $\mathcal{R}$ and $\mathcal{K}$, explaining how it illustrates some more general features about which we prove a basic theorem on sheared algebra maps. Maps from $\mathcal{R}$ to $\mathcal{K}$ are then the first application of the general theorem.

We shall begin with a look at the Dyer-Lashof algebra $\mathcal{R}$. Let $Y$ be an infinite loop space and $y \in H_{k}(Y)$. When one defines an action on $H_{k}(Y)$ by the conversion formula

$$
Q^{i}(y)=D_{i-k} y
$$

it is one of the minor miracles referred to in the introduction that this extends to produce a well-defined action of a bialgebra $\mathcal{R}$ on $H_{*}(Y)$; neither the relations forced on the $Q^{j}$ by those in $\mathcal{K}$ nor the coproduct formula in $\mathcal{K}$ become entangled, as should be feared, with the dimension $k$ of $y$ [May1, May2, DL]. More generally, any unstable $\mathcal{K}$-algebra produces an unstable $\mathcal{R}$-algebra and vice versa. In formal power series the conversion is

$$
Q(r) y(t)=D(r) y(r t)
$$

with $y(t)=\sum y_{k} t^{k}$ and $|r|=|t|=-1$.

To be more specific, the conversion first produces a bigraded $\mathbf{F}_{2}$-bialgebra $\mathcal{R}(-\infty)$ (we follow the notation of [CLM, Mill], and also call this the "algebraic" Dyer-Lashof algebra KL1] $)$. The algebra $\mathcal{R}(-\infty)$ is generated by $Q^{i} \in \mathcal{R}(-\infty)_{1, i}$ for $i \geq 0$, subject to Adem relations

$$
Q^{i} Q^{j}=\sum_{\frac{i}{2} \leq t \leq i-j-1}\left(\begin{array}{c}
t-j-1 \\
2 t-i
\end{array}\right) Q^{i+j-t} Q^{t} \quad \text { for } i>2 j,
$$

and with coproduct given by $\Delta Q^{i}=\sum Q^{t} \otimes Q^{i-t}$ May2.

We call a nonnegative sequence $I=\left(i_{1}, i_{2}, \ldots, i_{n}\right)$, and the corresponding monomial $Q^{I}=Q^{i_{1}} \cdots Q^{i_{n}}$, admissible, provided $i_{t} \leq 2 i_{t+1}$ for each $t$, and recall CLM, Mill that the admissible monomials form a basis for $\mathcal{R}(-\infty)$.

The excess of an admissible $I$ and of $Q^{I}$ is defined to be $i_{1}-\left(i_{2}+\cdots+i_{n}\right)$, and we recall that an admissible vanishes on all $k$-dimensional homology classes precisely if it has excess less than $k$ [CLM] p. 17]. Following [CLM, Mill, we consider the ideal $\mathcal{B}(k)$ in $\mathcal{R}(-\infty)$ spanned by all admissibles of excess less than $k$, and form the bigraded algebras $\mathcal{R}(k)=\mathcal{R}(-\infty) / \mathcal{B}(k)$, with basis the admissibles of excess at least $k$. Then $\mathcal{R}$, the "geometric" Dyer-Lashof algebra, is defined to be $\mathcal{R}(0)$, a quotient bialgebra of $\mathcal{R}(-\infty)$ [CLM, p. 17].

Before proceeding, we recall from section 2 the shift map $\delta: \mathcal{R}(-\infty)_{n, i} \rightarrow$ $\mathcal{R}(-\infty)_{n, i-\left(2^{n}-1\right)}$ defined by

$$
\delta\left(Q^{i_{1}} \cdots Q^{i_{n-1}} Q^{i_{n}}\right)=Q^{i_{1}-2^{n-1}} \cdots Q^{i_{n-1}-2} Q^{i_{n}-1}
$$

on admissibles. Note that $\delta$ reduces excess by one.

For each fixed $k \geq 0$, we inductively extend the conversion formula above for the action on $k$-dimensional classes, and abstract from this conversion on admissibles 
to define a map

$$
\hat{\theta}_{k}: \mathcal{R}(-\infty)_{n, i} \rightarrow \mathcal{K}_{n, i-\left(2^{n}-1\right) k}
$$

of vector spaces, commuting with the respective actions on $H_{k}(Y)$, given by the formula

$$
\hat{\theta}_{k}\left(Q^{i_{1}} \cdots Q^{i_{n-1}} Q^{i_{n}}\right)=D_{i_{1}-i_{2}-\cdots-i_{n}-k} \cdots D_{i_{n-1}-i_{n}-k} D_{i_{n}-k} .
$$

Note that at this time we only know that this formula for $\hat{\theta}_{k}$ holds on admissibles.

From our remarks above we know that $\hat{\theta}_{k}$ factors through $\mathcal{R}(k)$, creating

$$
\theta_{k}: \mathcal{R}(k)_{n, i} \rightarrow \mathcal{K}_{n, i-\left(2^{n}-1\right) k}
$$

satisfying the same formula on admissibles, from which it is easily verified that

Proposition 5.1. For each $k \geq 0$,

(i) $\theta_{k+1}=\alpha \circ \theta_{k} \quad$ and $\quad \theta_{k+1}=\theta_{k} \circ \delta$;

(ii) $\theta_{k}$ is an isomorphism of vector spaces; and

(iii) $\theta_{0}$ is an isomorphism of coalgebras.

It follows that the dual of $\theta_{0}$ is an algebra isomorphism. Combining this fact with Theorem [B] yields Madsen's theorem [Mad] on the algebra structure of $\mathcal{R}^{*}$. Further combining with Theorem[E gives us Madsen's computation of the $\mathcal{A}$-action (of the $S q^{2^{l}}$ ) on generators of the Dickson algebras $\mathcal{R}_{n}^{*}$.

The bijections $\theta_{k}$ will be some of our first examples of "sheared" algebra maps. While not algebra maps in the traditional sense, we see that they respect products in a sheared sense (at least on admissible monomials), using the shift map $\delta$. For example, while $\theta_{k}$ maps $Q^{i}$ to $D_{i-k}$, it maps an admissible $Q^{i_{1}} Q^{i_{2}}$ to $D_{i_{1}-i_{2}-k} D_{i_{1}-k}=\theta_{k}\left(\delta^{i_{2}}\left(Q^{i_{1}}\right)\right) \theta_{k}\left(Q^{i_{2}}\right)$. Thus as $\theta_{k}$ passes through the product it is sheared by a shifting of the left factor. The shifting is by $i_{2}$ applications of the shift map $\delta$, where $i_{2}$ is the topological degree of the right factor. This is reminiscent in some respects of the notion of a semidirect product of groups.

We will show that this phenomenon has both general structure and application. A sheared algebra map should behave as above on any product, not just on monomials of special form like admissibles. This is a pleasant circumstance, since it means the entire feature is compatible with (Adem) relations, and thus it is a general property of the map, unaffected by the representation of elements. In particular, the formula we gave above for $\hat{\theta}_{k}$ will hold, even on inadmissibles!

With these motivating examples in hand, we now proceed to describe the general setting for sheared algebra maps, and prove a theorem which can be applied to show that several of our constructions produce sheared algebra bijections. In addition to the maps $\theta_{k}: \mathcal{R}(k) \rightarrow \mathcal{K}$, these applications will include a map relating the cohomology of $\mathcal{K}$ and the Steenrod algebra, i.e. a sheared algebra bijection $H^{*}(\mathcal{K}) \rightarrow \mathcal{A}$. This will arise from sheared algebra maps involving a new graded algebra $\mathcal{K}(\infty)$ which we will construct from $\mathcal{K}$.

First, we recall from Definition 2.4 what is meant by a sheared algebra homomorphism.

Our general method for producing sheared algebra homomorphisms requires $K$ to be a Poincaré-Birkhoff-Witt algebra (P-B-W algebra for short), about which we briefly remind the reader, and refer to $[\mathrm{Pr}]$ or $[\mathrm{Lo}]$. The Lie Steenrod algebra and algebraic Dyer-Lashof algebra are examples [Lo, $[\mathrm{Mill}, \overline{\mathrm{Pr}}]$, as well as our $\mathcal{K}$ and some other algebras we will encounter. 
Begin with an augmented algebra $K$ with generating set $\hat{K}=\left\{b_{i}\right\}_{i \in \mathcal{I}}, \mathcal{I}$ a subset of $\mathbb{Z}^{+}$. Suppose further that $K$ has only homogeneous 2 -fold relations in these generators. Then $K$ is called a homogeneous pre-Koszul algebra, and has a multiplicative length grading, which we denote by $K_{*}$. (We are being somewhat more general than $[\mathrm{Lo}, \overline{\mathrm{Pr}}]$ by not yet considering or requiring a second, topological, grading.)

Let $I=\left(i_{1}, \ldots, i_{n}\right)$ denote a multi-index and call it the label of $b_{I}=b_{i_{1}} \cdots b_{i_{n}}$, the corresponding monomial in generators. Suppose $S$ is a set of multi-indices chosen so that $\mathcal{B}=\left\{b_{I}\right\}_{I \in S}$ is a vector space basis for $K$. Then $(\mathcal{B}, S)$ is called a labeled basis for $K$. The monomials in $\mathcal{B}$ are called admissibles, the expression of any element in terms of them is called its admissible expression, and the admissible expression of any inadmissible 2-fold product of generators is called an admissible relation. Now the set of all possible multi-indices is ordered, first by length and then lexicographically (using the natural ordering on $\mathbb{Z}^{+}$). The labeled basis $(\mathcal{B}, S)$ is called a $P-B-W$ basis (and then $(K, \hat{K}, \mathcal{B}, S)$ a $P-B-W$ algebra) provided:

1. If $I, J \in S$, then either the juxtaposition $(I, J)$ is also in $S$ or else the label of every monomial in the admissible expression for $b_{I} b_{J}$ is strictly less than $(I, J)$, and

2. For $n>2,\left(i_{1}, \ldots, i_{n}\right) \in S$ if and only if for each $j<n$ we have $\left(i_{1}, \ldots, i_{j}\right) \in S$ and $\left(i_{j+1}, \ldots, i_{n}\right) \in S$.

Now we will describe a process which begins with a P-B-W algebra $K$, and constructs under three assumptions a particular type of degree function $d$ and shift map $F$ which interact nicely. The resulting $(K, \hat{K}, F, d)$ will be called an "algebra with shifting", we then prove a theorem enabling the creation of various sheared algebra homomorphisms with $K$ as domain. We will apply this theorem first to $\hat{\theta}_{k}: \mathcal{R}(-\infty) \rightarrow \mathcal{K}$, and then show that each $\theta_{k}: \mathcal{R}(k) \rightarrow \mathcal{K}$ is also a sheared algebra bijection. We will later apply the theorem in several other situations.

So we start with a P-B-W algebra $(K, \hat{K}, \mathcal{B}, S)$ and describe first the construction of a degree function $d$ under

Assumption 1. Suppose $b$ is a positive integer and suppose $d: \hat{K} \rightarrow \mathbb{Z}^{+}$is given satisfying the following condition: If $k^{\prime} k^{\prime \prime}=\sum l_{m}^{\prime} l_{m}^{\prime \prime}$ is an admissible relation, then for each $m$

$$
d\left(k^{\prime}\right)+b d\left(k^{\prime \prime}\right)=d\left(l_{m}^{\prime}\right)+b d\left(l_{m}^{\prime \prime}\right) .
$$

Examples. For $b=1$, any additively graded algebra, e.g. topological degree in $\mathcal{R}(-\infty)$, the negative of topological degree in $\mathcal{A}$ (i.e., positively graded), or length degree in $\mathcal{R}(-\infty)$ or $\mathcal{K}$ or $\mathcal{A}_{L}$ (the Lie Steenrod algebra [ $\left.\mathrm{Lo}, \operatorname{\mathrm {Pr}}\right]$ ). For $b=2$, topological degree in $\mathcal{K}$. $\hat{K}$.

To extend $d$ to all monomials in $K$, consider first $T(\hat{K})$, the tensor algebra on

Definition 5.2. On the usual basis for $T(\hat{K})$ define

$$
\hat{d}\left(k_{1} \otimes \cdots \otimes k_{n}\right)=d\left(k_{1}\right)+b d\left(k_{2}\right)+b^{2} d\left(k_{3}\right)+\cdots+b^{n-1} d\left(k_{n}\right) .
$$

Lemma 5.3. If $k_{i} k_{i+1}=\sum l_{m}^{\prime} l_{m}^{\prime \prime}$ is an admissible relation, then for each $m$

$$
\hat{d}\left(k_{1} \otimes \cdots \otimes k_{i} \otimes k_{i+1} \otimes \cdots \otimes k_{n}\right)=\hat{d}\left(k_{1} \otimes \cdots \otimes l_{m}^{\prime} \otimes l_{m}^{\prime \prime} \otimes \cdots \otimes k_{n}\right) .
$$


Proof. We need only check that

$$
b^{i-1} d\left(k_{i}\right)+b^{i} d\left(k_{i+1}\right)=b^{i-1} d\left(l_{m}^{\prime}\right)+b^{i} d\left(l_{m}^{\prime \prime}\right),
$$

which follows from $d\left(k_{i}\right)+b d\left(k_{i+1}\right)=d\left(l_{m}^{\prime}\right)+b d\left(l_{m}^{\prime \prime}\right)$.

Corollary 5.4. If we define a map $d$ on a typical monomial (of the form $k_{1} \cdots k_{n}$ where each $\left.k_{i} \in \hat{K}\right)$ by the formula $d\left(k_{1} \cdots k_{n}\right)=\hat{d}\left(k_{1} \otimes \cdots \otimes k_{n}\right)$, then this yields a well-defined $d$ on each element of $K$ which can be expressed as a monomial, in the sense that if $k_{1} \cdots k_{n}=\sum l_{1 m} \cdots l_{n m}$ expresses $k_{1} \cdots k_{n}$ as a sum of admissibles, then for each $m, d\left(k_{1} \cdots k_{n}\right)=d\left(l_{1 m} \cdots l_{n m}\right)$.

Remark 5.5. Thus $d$ of a monomial is independent of the various possible monomial representations of the element, even though $d$ is defined and calculated from any monomial representation.

We now state the main fact concerning $d$, whose easy proof we leave to the reader:

Theorem 5.6. If $z$ and $z^{\prime}$ are monomials in $K$ with $z \in K_{m}$, then $d\left(z z^{\prime}\right)=$ $d(z)+b^{m} d\left(z^{\prime}\right)$.

Thus the degree $d$ is in general "skew-additive" unless $b=1$, which is the traditional additivity on products.

Note that the algebra $K_{*, *}$ is now bigraded via length and degree.

We next describe the construction of a shift map $F$, a vector space endomorphism of $K$, under

Assumption 2. Suppose $a$ is a positive integer, and suppose a map $F: \hat{K} \rightarrow$ $\hat{K} \cup\{0\}$ is given satisfying the condition: If $k^{\prime} k^{\prime \prime}=\sum l_{m}^{\prime} l_{m}^{\prime \prime}$ is an admissible relation, then

$$
F^{a}\left(k^{\prime}\right) F\left(k^{\prime \prime}\right)=\sum F^{a}\left(l_{m}^{\prime}\right) F\left(l_{m}^{\prime \prime}\right)
$$

is also an admissible relation.

Examples. For $a=1, K$ any P-B-W algebra with $F$ the restriction to $\hat{K}$ of any algebra endomorphism that takes generators to generators or to 0 (e.g., $\mathcal{K}$ with $\alpha$ ). For $a=2, \mathcal{R}(-\infty)$ with $F=\delta$.

Definition 5.7. Define a linear transformation $\hat{F}: T(\hat{K}) \rightarrow K$ by setting

$$
\hat{F}\left(k_{1} \otimes \cdots \otimes k_{n}\right)=F^{a^{n-1}}\left(k_{1}\right) \cdots F^{a}\left(k_{n-1}\right) F\left(k_{n}\right)
$$

and

$$
\hat{F}(1)=1
$$

on the usual basis elements.

Lemma 5.8. If $k_{i} k_{i+1}=\sum l_{m}^{\prime} l_{m}^{\prime \prime}$ is an admissible relation, then

$$
\hat{F}\left(k_{1} \otimes \cdots \otimes k_{i} \otimes k_{i+1} \otimes \cdots \otimes k_{n}\right)=\sum \hat{F}\left(k_{1} \otimes \cdots \otimes l_{m}^{\prime} \otimes l_{m}^{\prime \prime} \otimes \cdots \otimes k_{n}\right) .
$$

Proof. We begin by noting that for any positive integer $e$,

$$
F^{e a}\left(k_{i}\right) F^{e}\left(k_{i+1}\right)=\sum F^{e a}\left(l_{m}^{\prime}\right) F^{e}\left(l_{m}^{\prime \prime}\right)
$$


We compute

$$
\begin{aligned}
\hat{F}\left(k_{1}\right. & \left.\otimes \cdots \otimes k_{i} \otimes k_{i+1} \otimes \cdots \otimes k_{n}\right) \\
& =F^{a^{n-1}}\left(k_{1}\right) \cdots F^{a^{n-i+1}}\left(k_{i-1}\right) F^{a^{n-i}}\left(k_{i}\right) \cdots F^{a}\left(k_{n-1}\right) F\left(k_{n}\right) .
\end{aligned}
$$

On the other hand,

$$
\begin{aligned}
& \sum \hat{F}\left(k_{1} \otimes \cdots \otimes l_{m}^{\prime} \otimes l_{m}^{\prime \prime} \otimes \cdots \otimes k_{n}\right) \\
& \quad=\sum F^{a^{n-1}}\left(k_{1}\right) \cdots F^{a^{n-i+1}}\left(l_{m}^{\prime}\right) F^{a^{n-i}}\left(l_{m}^{\prime \prime}\right) \cdots F^{a}\left(k_{n-1}\right) F\left(k_{n}\right) .
\end{aligned}
$$

Hence the proof is completed by checking that

$$
F^{a^{n-i+1}}\left(k_{i-1}\right) F^{a^{n-i}}\left(k_{i}\right)=\sum F^{a^{n-i+1}}\left(l_{m}^{\prime}\right) F^{a^{n-i}}\left(l_{m}^{\prime \prime}\right),
$$

which follows from the remark at the beginning of the proof, taking $e=a^{n-i}$.

Corollary 5.9. We can extend $F$ to an endomorphism of $K$ by the formula

$$
F\left(k_{1} \cdots k_{n}\right)=\hat{F}\left(k_{1} \otimes \cdots \otimes k_{n}\right) .
$$

In particular, $F$ is well defined on the set of monomials in $K$ using the formula from Definition 5.7, in the sense that if $k_{1} \cdots k_{n}=\sum l_{1 m} \cdots l_{n m}$ expresses $k_{1} \cdots k_{n}$ as a sum of admissibles, then $F\left(k_{1} \cdots k_{n}\right)=\sum F\left(l_{1 m} \cdots l_{n m}\right)$.

Corollary 5.10. For each positive integer e,

$$
F^{e}\left(k_{1} \cdots k_{n}\right)=F^{a^{n-1} e}\left(k_{1}\right) \cdots F^{e}\left(k_{n}\right) .
$$

From the above, we obtain the main theorem concerning $F$.

Theorem 5.11. If $e$ is a positive integer and $z_{1}$ and $z_{2}$ are monomials with $z_{2} \in$ $K_{n, *}$, then

$$
F^{e}\left(z_{1} z_{2}\right)=F^{a^{n} e}\left(z_{1}\right) F^{e}\left(z_{2}\right) .
$$

Assume now that $d$ and $F$ have been defined, as above, using Assumptions [1] and 2. Suppose, in addition, that they interact as in

Assumption 3. For $k \in \hat{K}$,

$$
d(F(k))=d(k)+b-a .
$$

Then we are prepared for

Definition 5.12. Let $K$ be a P-B-W algebra satisfying Assumptions 1, 2, 3 with shift map $F$ and (skew-additive) degree $d$ as above. We call the quadruple $(K, \hat{K}, F, d)$ an algebra with shifting.

Examples. Any additively graded algebra with $a=b=1, F$ the identity, and $d$ the grading. Also, $\mathcal{R}(-\infty)$ with $a=2, b=1, F=\delta$, and $d$ the topological degree. We will have two other important examples soon.

Remark 5.13. While $\mathcal{K}$ has both degree and shifting maps, they do not interact as in Assumption 3 .

Theorem 5.14. Let $(K, \hat{K}, F, d)$ be an algebra with shifting. If $z$ is a monomial in $K_{n, *}$, then

$$
d(F(z))=d(z)+b^{n}-a^{n} .
$$


Proof. Write $z$ in monomial form $k_{1} \cdots k_{n}$. We compute

$$
\begin{aligned}
d\left(F\left(k_{1} \cdots k_{n}\right)\right)= & d\left(F^{a^{n-1}}\left(k_{1}\right) \cdots F^{a}\left(k_{n-1}\right) F\left(k_{n}\right)\right) \\
= & d\left(F^{a^{n-1}}\left(k_{1}\right)\right)+b d\left(F^{a^{n-2}}\left(k_{2}\right)\right)+\ldots \\
& +b^{n-2} d\left(F^{a}\left(k_{n-1}\right)\right)+b^{n-1} d\left(F\left(k_{n}\right)\right) \\
= & \left(d\left(k_{1}\right)+a^{n-1}(b-a)\right)+b\left(d\left(k_{2}\right)+a^{n-2}(b-a)\right)+\cdots \\
& +b^{n-2}\left(d\left(k_{n-1}\right)+a(b-a)\right)+b^{n-1}\left(d\left(k_{n}\right)+b-a\right) \\
= & \left(d\left(k_{1}\right)+b d\left(k_{2}\right)+\cdots+b^{n-1} d\left(k_{n}\right)\right) \\
& +\left(a^{n-1}+a^{n-2} b+\cdots+a b^{n-2}+b^{n-1}\right)(b-a) \\
= & d\left(k_{1} \cdots k_{n}\right)+b^{n}-a^{n} .
\end{aligned}
$$

We are now ready to prove the main theorem on algebras with shifting, Theorem F. which will enable us to produce various sheared algebra homomorphisms. We shall begin the proof with two preparatory lemmas.

Lemma 5.15. Let e be a positive integer and let $k^{\prime} k^{\prime \prime}=\sum l_{m}^{\prime} l_{m}^{\prime \prime}$ be an admissible relation. Then under the hypotheses of the theorem,

$$
\psi\left(F^{d\left(k^{\prime \prime}\right)+b e}\left(k^{\prime}\right)\right) \psi\left(F^{e}\left(k^{\prime \prime}\right)\right)=\sum \psi\left(F^{d\left(l_{m}^{\prime \prime}\right)+b e}\left(l_{m}^{\prime}\right)\right) \psi\left(F^{e}\left(l_{m}^{\prime \prime}\right)\right)
$$

Proof. Using Assumption 3, we compute

$$
\begin{aligned}
\psi\left(F^{d\left(k^{\prime \prime}\right)+b e}\left(k^{\prime}\right)\right) \psi\left(F^{e}\left(k^{\prime \prime}\right)\right) \\
=\psi\left(F^{d\left(k^{\prime \prime}\right)+b e-a e}\left(F^{a e}\left(k^{\prime}\right)\right)\right) \psi\left(F^{e}\left(k^{\prime \prime}\right)\right) \\
=\psi\left(F^{d\left(F^{e}\left(k^{\prime \prime}\right)\right)}\left(F^{a e}\left(k^{\prime}\right)\right)\right) \psi\left(F^{e}\left(k^{\prime \prime}\right)\right) .
\end{aligned}
$$

By Assumption 2,

$$
F^{a e}\left(k^{\prime}\right) F^{e}\left(k^{\prime \prime}\right)=\sum F^{a e}\left(l_{m}^{\prime}\right) F^{e}\left(l_{m}^{\prime \prime}\right)
$$

is an admissible relation, so applying the hypothesis of the theorem, the last term above is

$$
\sum \psi\left(F^{d\left(F^{e}\left(l_{m}^{\prime \prime}\right)\right)} F^{a e}\left(l_{m}^{\prime}\right)\right) \psi\left(F^{e}\left(l_{m}^{\prime \prime}\right)\right)
$$

which by reversing the above steps is

$$
\sum \psi\left(F^{d\left(l_{m}^{\prime \prime}\right)+b e}\left(l_{m}^{\prime}\right)\right) \psi\left(F^{e}\left(l_{m}^{\prime \prime}\right)\right)
$$

as desired.

Lemma 5.16. For any monomial $k_{1} \cdots k_{n}$,

$$
\psi\left(k_{1} \cdots k_{n}\right)=\psi\left(F^{d\left(k_{2} \cdots k_{n}\right)}\left(k_{1}\right)\right) \cdots \psi\left(F^{d\left(k_{n}\right)}\left(k_{n-1}\right)\right) \psi\left(k_{n}\right) .
$$

Proof. Define $\hat{\psi}: T(\hat{K}) \rightarrow M$ by

$$
\hat{\psi}\left(k_{1} \otimes \cdots \otimes k_{n}\right)=\psi\left(F^{d\left(k_{2} \cdots k_{n}\right)}\left(k_{1}\right)\right) \cdots \psi\left(F^{d\left(k_{n}\right)}\left(k_{n-1}\right)\right) \psi\left(k_{n}\right) .
$$

From Lemma [5.15] Corollary [5.4 and Theorem [5.6] we see by taking $e=$ $d\left(k_{i+2} \cdots k_{n}\right)$, that if $k_{i} k_{i+1}=\sum l_{m}^{\prime} l_{m}^{\prime \prime}$ is an admissible relation, then

$$
\begin{aligned}
\hat{\psi}\left(k_{1}\right. & \left.\otimes \cdots \otimes k_{i} \otimes k_{i+1} \otimes \cdots \otimes k_{n}\right) \\
& =\sum \hat{\psi}\left(k_{1} \otimes \cdots \otimes l_{m}^{\prime} \otimes l_{m}^{\prime \prime} \otimes \cdots \otimes k_{n}\right) .
\end{aligned}
$$


Proof of Theorem E. Let $z=k_{1} \cdots k_{m}$ and $z^{\prime}=k_{1}^{\prime} \cdots k_{n}^{\prime}$ be monomials. Then

$$
\begin{array}{r}
\psi\left(z z^{\prime}\right)=\psi\left(k_{1} \cdots k_{m} z^{\prime}\right)=\psi\left(F^{d\left(k_{2} \cdots k_{m} z^{\prime}\right)}\left(k_{1}\right)\right) \cdots \psi\left(F^{d\left(k_{i+1} \cdots k_{m} z^{\prime}\right)}\left(k_{i}\right)\right) \cdots \\
\psi\left(F^{d\left(z^{\prime}\right)}\left(k_{m}\right)\right) \psi\left(z^{\prime}\right) \quad(\text { Lemma 5.16). }
\end{array}
$$

For each $i$, write $f_{i}=F^{a^{m-i} d\left(z^{\prime}\right)}\left(k_{i}\right)$. Now, we also compute

$$
\begin{aligned}
& \psi\left(F^{d\left(z^{\prime}\right)}(z)\right)=\psi\left(F^{d\left(z^{\prime}\right)}\left(k_{1} \cdots k_{m}\right)\right) \\
& =\psi\left(F^{a^{m-1} d\left(z^{\prime}\right)}\left(k_{1}\right) \cdots F^{a^{m-i} d\left(z^{\prime}\right)}\left(k_{i}\right) \cdots F^{d\left(z^{\prime}\right)}\left(k_{m}\right)\right) \quad\left(\text { Cor. } 5.10 \text { with } e=d\left(z^{\prime}\right)\right) \\
& =\psi\left(f_{1} \cdots f_{i} \cdots f_{m}\right) \\
& =\psi\left(F^{d\left(f_{2} \cdots f_{m}\right)}\left(f_{1}\right)\right) \cdots \psi\left(F^{d\left(f_{i+1} \cdots f_{m}\right)}\left(f_{i}\right)\right) \cdots \psi\left(f_{m}\right) \quad \text { (Lemma 5.16). }
\end{aligned}
$$

So to finish the proof, it will suffice to show that for each $i$

$$
F^{d\left(f_{i+1} \cdots f_{m}\right)}\left(f_{i}\right)=F^{d\left(k_{i+1} \cdots k_{m} z^{\prime}\right)}\left(k_{i}\right),
$$

i.e., that

$$
F^{d\left(f_{i+1} \cdots f_{m}\right)}\left(F^{a^{m-i}} d\left(z^{\prime}\right)\left(k_{i}\right)\right)=F^{d\left(k_{i+1} \cdots k_{m} z^{\prime}\right)}\left(k_{i}\right),
$$

which amounts to showing that

$$
d\left(f_{i+1} \cdots f_{m}\right)+a^{m-i} d\left(z^{\prime}\right)=d\left(k_{i+1} \cdots k_{m} z^{\prime}\right) .
$$

We have

$$
\begin{aligned}
d\left(f_{i+1}\right. & \left.\cdots f_{m}\right)+a^{m-i} d\left(z^{\prime}\right) \\
= & d\left(F^{a^{m-(i+1)} d\left(z^{\prime}\right)}\left(k_{i+1}\right) \cdots F^{d\left(z^{\prime}\right)}\left(k_{m}\right)\right)+a^{m-i} d\left(z^{\prime}\right) \\
= & d\left(F^{d\left(z^{\prime}\right)}\left(k_{i+1} \cdots k_{m}\right)\right)+a^{m-i} d\left(z^{\prime}\right) \quad(\text { Corollary 5.10) } \\
= & d\left(k_{i+1} \cdots k_{m}\right)+\left(b^{m-i}-a^{m-i}\right) d\left(z^{\prime}\right)+a^{m-i} d\left(z^{\prime}\right) \quad(\mathrm{T} \\
= & d\left(k_{i+1} \cdots k_{m}\right)+b^{m-i} d\left(z^{\prime}\right) \\
= & d\left(k_{i+1} \cdots k_{m} z^{\prime}\right) \quad(\text { Theorem 5.6). }
\end{aligned}
$$

As promised, we can now immediately apply Theorem $\mathrm{F}$ to prove the following theorem, which includes Theorem $\mathrm{G}$ as a special case.

Theorem 5.17. For $k \geq 0, \hat{\theta}_{k}: \mathcal{R}(-\infty) \rightarrow \mathcal{K}$ is a sheared algebra homomorphism, and $\theta_{k}: \mathcal{R}(k) \rightarrow \mathcal{K}$ is a sheared algebra bijection. In particular, the defining formula for $\hat{\theta}_{k}$ holds on arbitrary products, not just admissibles.

Proof. $\mathcal{R}(-\infty)$ is a P-B-W algebra, and an algebra with shifting using $d=$ topological degree with $b=1$ and $F=\delta$ with $a=2$, since

$$
d\left(\delta\left(Q^{i}\right)\right)=d\left(Q^{i-1}\right)=i-1=d\left(Q^{i}\right)+b-a,
$$

verifying Assumption 3 . Thus we can apply Theorem $\mathrm{F}$ by first defining $\hat{\theta}_{k}\left(Q^{i}\right)=$ $D_{i-k}$ and then verifying that Adem relations are respected, as required by the hypothesis of the theorem, which is a straightforward calculation. The earlier factorization of $\hat{\theta}_{k}$ through $\mathcal{R}(k)$ then yields the claim that $\theta_{k}$ is a sheared algebra map (since $d$ and $\delta$ both pass to the quotient $\mathcal{R}(k)$, even without needing to know whether $\mathcal{R}(k)$ is a $\mathrm{P}-\mathrm{B}-\mathrm{W}$ algebra or algebra with shifting). We have seen earlier in Proposition [5.1 that each $\theta_{k}$ is a bijection. 
Remark 5.18. Dualizing Theorem 5.17 for $k=0$ will shed light on the similarities and differences between the coproduct formulae in $\mathcal{K}^{*}$ and $\mathcal{R}^{*}$, since $\theta_{0}^{*}: \mathcal{K}^{*} \rightarrow \mathcal{R}^{*}$ is an algebra isomorphism (Proposition 5.1) and a co-sheared coalgebra bijection (Theorem 5.17). We will label elements of $\mathcal{R}^{*}$ using our description of $\mathcal{K}^{*}$ (Theorem B). Our defining formulas show that $\delta$ and $\alpha$ correspond under $\theta_{k}$, i.e. $\theta_{k} \circ \delta=\alpha \circ \theta_{k}$, so we have the shearing formula

$$
\theta_{0} \circ \mu_{\mathcal{R}}=\mu_{\mathcal{K}} \circ\left(\theta_{0} \otimes \theta_{0}\right) \circ \gamma=\mu_{\mathcal{K}} \circ \beta \circ\left(\theta_{0} \otimes \theta_{0}\right),
$$

where $\gamma\left(z \otimes z^{\prime}\right)=\delta^{d\left(z^{\prime}\right)}(z) \otimes z^{\prime}$ and $\beta\left(y \otimes y^{\prime}\right)=\alpha^{d\left(y^{\prime}\right)}(y) \otimes y^{\prime}$. Since $\alpha$ is dual to multiplication by $\chi$ in $\mathcal{K}^{*}$ (Example [3.18) we can dualize, beginning with our formula (Theorem C)

$$
\Delta_{\mathcal{K}^{*}}\left(x_{i, j}\right)=\sum_{p, q} x_{i-p, j-q}^{2^{p}} \otimes x_{p, q}
$$

obtaining

$$
\begin{array}{r}
\Delta_{\mathcal{R}^{*}}\left(x_{i, j}\right)=\sum_{p, q} \chi^{2^{p}\left(2^{q}-1\right)} x_{i-p, j-q}^{2^{p}} \otimes x_{p, q} \\
=\sum_{p, q} x_{0, i+j-p-q}^{2^{p}\left(2^{q}-1\right)} x_{i-p, j-q}^{2^{p}} \otimes x_{p, q} .
\end{array}
$$

This recovers Madsen's formula $\mathrm{Mad}$ for the coproduct in $\mathcal{R}^{*}$. We see that the extra complicating factors $x_{0, i+j-p-q}^{2^{p}\left(2^{q}-1\right)}$ in the coproduct in $\mathcal{R}^{*}$ are precisely those induced by the coshearing. The simpler coproduct in $\mathcal{K}^{*}$ is one of the reasons why $\mathcal{K}$ may prove more useful than $\mathcal{R}$ in some applications.

\section{Relationship Between $\mathcal{K}$ and the Steenrod algebra}

As mentioned in section 2, while relating $\mathcal{K}$ to $\mathcal{R}$ was quite easy to accomplish, it is more challenging to relate $\mathcal{K}$ to the Steenrod algebra $\mathcal{A}$ via a sheared algebra bijection, but this is our aim. Let $X$ be a space, and grade cohomology negatively, as before (Remark 3.5). Let $x \in H^{-k}(X), k \geq 0$. When one defines the action of $\mathcal{A}$ on $H^{-k}(X)$ by the conversion formula

$$
S q^{j}(x)=D_{k-j}(x),
$$

another minor miracle occurs and this produces a well-defined action of a Hopf algebra $\mathcal{A}$ on $H^{*}(X)$ May1, Stee3. As in the previous section, any unstable algebra over $\mathcal{K}$ produces an unstable $\mathcal{A}$-algebra and vice versa. We will also grade the Steenrod algebra $\mathcal{A}$ negatively. In formal power series we have

$$
S q(r) x(t)=D\left(r^{-1}\right) x(r t)
$$

with $|r|=|t|=1$ (and the contragredient homology action

$$
S q_{*}(r) y(t)=D_{*}\left(r^{-\frac{1}{2}}\right) y\left(r^{\frac{1}{2}} t\right)
$$

with $|r|=1,|t|=-1)$.

As we did before with $\mathcal{R}(-\infty)$, for each $k \geq 0$ we inductively extend the conversion formula and abstract from it on admissibles to produce a map, commuting with the actions on $H^{*}(X)$,

$$
\phi_{k}: \mathcal{A} \rightarrow \mathcal{K},
$$


given on an admissible monomial $\left(a_{l-1} \geq 2 a_{l}>0\right)$ by the formula

$$
\phi_{k}\left(S q^{a_{1}} \cdots S q^{a_{n}}\right)=D_{b_{1}} \cdots D_{b_{n}} \in \mathcal{K}_{n,\left(2^{n}-1\right) k-j}
$$

where $S q^{a_{1}} \cdots S q^{a_{n}} \in \mathcal{A}^{-j}$ and

$$
b_{l}=\left(k+a_{n}+\cdots+a_{l+1}\right)-a_{l} .
$$

Remark 6.1. Some words of warning: It is curious that while this general conversion formula is correct even on inadmissibles when used to compare the two actions on $H^{k} X$, it is nonetheless false for $\phi_{k}$ itself. For instance, while $\phi_{4}\left(S q^{1} S q^{2}\right)=$ $\phi_{4}\left(S q^{3}\right)=D_{1}, S q^{1} S q^{2} x_{4}=D_{5} D_{2} x_{4}$, so $D_{1}$ and $D_{5} D_{2}$ agree on every 4-dimensional class even though $D_{1} \neq D_{5} D_{2}$, and the formula for $\phi_{4}$ defined above does not hold on $S q^{1} S q^{2}$, even though it does hold when followed by application to a cohomology class. This is due to the identification of $S q^{0}$ with 1 in the Steenrod algebra for topological reasons, which, in particular, prevents the Adem relations from being homogeneous, so $\mathcal{A}$ possesses no length grading. As a slightly different and more perplexing warning example, $S q^{1} S q^{3}=0$, but $S q^{1} S q^{3} x_{6}$ converts to $D_{8} D_{3} x_{6}$. Now $D_{8} D_{3}=D_{0} D_{7}$, which is nonzero in $\mathcal{K}$, but of course is zero on every 6 -dimensional class. This more subtle type of conversion failure can be avoided if one can arrange to encounter only "stable" Adem relations $S q^{a} S q^{b}=\cdots$, those for which $b \leq a<$ $2 b$; they exhibit several very useful patterns and features. We could develop our analysis in this direction, but will take a different tack since in the end we will need to reverse the correspondence, ultimately mapping to, instead of from, the Steenrod algebra, with a sheared algebra bijection. This illustrates the noninvertibility of sheared algebra bijections.

Despite the caution and pessimism advised by this remark, we intend to fit the $\phi_{k}$ together to obtain a close relationship between $\mathcal{A}$ and $\mathcal{K}$, noting first that it is for $k$ large (quite contrary to $\theta_{0}: \mathcal{R} \cong \mathcal{K}$ ) that we should expect $\phi_{k}$ to match $\mathcal{A}$ well with $\mathcal{K}$. Given the warnings above, one might expect there will be no general map relating the multiplications in $\mathcal{A}$ and $\mathcal{K}$ in sheared fashion, but we will find, surprisingly, that there is.

Recall from Definition 2.6 that $\mathcal{K}(k)$ is defined to be the vector subspace of $\mathcal{K}$ spanned by the admissible monomials of the form $D_{0}^{i_{0}} D_{1}^{i_{1}} \cdots D_{k-1}^{i_{k-1}}$. We have

\section{Proposition 6.2.}

(i) $\mathcal{K}(k)$ is a sub-bialgebra of $\mathcal{K}$; and

(ii) there is an exact sequence of vector spaces

$$
0 \rightarrow \mathcal{K}(k) \stackrel{\kappa}{\rightarrow} \mathcal{K}(k) \stackrel{\alpha}{\rightarrow} \mathcal{K}(k-1) \rightarrow 0
$$

Proof. Immediate from the definitions. (Recall the maps $\alpha$ and $\kappa$ from section 3])

The following proposition lists some information about $\phi_{k}$.

\section{Proposition 6.3.}

(i) The image of $\phi_{k}$ is $\mathcal{K}(k)$.

(ii) The kernel of $\phi_{k}$ is the span of the admissible monomials in $\mathcal{A}$ with excess greater than $k$.

(iii) $\phi_{k-1}=\alpha \circ \phi_{k}$. 
Proof. Let $S q^{A}=S q^{a_{1}} \cdots S q^{a_{n}}$ be an admissible monomial in $\mathcal{A}$, i.e. $a_{l-1} \geq 2 a_{l}>$ 0 . Since $a_{n}>0, b_{n}=k-a_{n}<k$. Furthermore,

$$
\begin{aligned}
b_{l}-b_{l-1} & =\left[\left(k+a_{n}+\cdots+a_{l+1}\right)-a_{l}\right]-\left[\left(k+a_{n}+\cdots+a_{l}\right)-a_{l-1}\right] \\
& =a_{l-1}-2 a_{l} \geq 0 .
\end{aligned}
$$

So $\phi_{k}$ takes admissibles to admissibles, and $\operatorname{Im}\left(\phi_{k}\right) \subseteq \mathcal{K}(k)$. Now suppose that the excess of $S q^{A}$ is less than $k+1$. Then

$$
\begin{aligned}
b_{1} & =k+\left(a_{n}+\cdots+a_{2}\right)-a_{1} \\
& =k-\left[a_{1}-\left(a_{n}+\cdots+a_{2}\right)\right] \\
& =k-\operatorname{excess}\left(S q^{A}\right) \geq 0 .
\end{aligned}
$$

Since the $n$-tuple $\left(b_{1}, \ldots, b_{n}\right)$ completely determines $\left(a_{1}, \ldots, a_{n}\right)$, the restriction of $\phi_{k}$ to the space of admissibles of excess $\leq k$ is an isomorphism. Finally, if the excess of $S q^{A}$ is greater than $k$, then the preceding calculation shows that $b_{1}<0$, i.e. that $D_{b_{1}}=0$. This proves part (ii). Part (iii) is immediate from the definitions.

Definition 6.4. We define $\mathcal{K}(\infty)_{*, *}$ by

$$
\mathcal{K}(\infty)_{n,-i}={\underset{\leftarrow}{k}}_{\lim } \mathcal{K}(k)_{n,\left(2^{n}-1\right) k-i} \quad \text { for } n, i \geq 0
$$

under the maps $\alpha$ (recalling that $\left.\alpha: \mathcal{K}(k)_{n,\left(2^{n}-1\right) k-i} \rightarrow \mathcal{K}(k-1)_{n,\left(2^{n}-1\right)(k-1)-i}\right)$.

Note $\mathcal{K}(\infty)_{*, *}$ has negative topological grading. The products in the $\mathcal{K}(k)$ combine under the bonding algebra maps $\alpha$ to make $\mathcal{K}(\infty)_{*, *}$ an algebra with length and topological degrees behaving as in $\mathcal{K}$ :

$$
\mu_{\infty}: \mathcal{K}(\infty)_{m, i} \otimes \mathcal{K}(\infty)_{n, j} \rightarrow \mathcal{K}(\infty)_{m+n, i+2^{m} j} .
$$

Theorem 6.5. The algebra $\mathcal{K}(\infty)$ is described by generators $D_{\infty-i} \in \mathcal{K}(\infty)_{1,-i}$, for $i>0$, with relations for $i<j$

$$
D_{\infty-i} D_{\infty-j}=\sum\left(\begin{array}{c}
j-p-1 \\
i+j-2 p
\end{array}\right) D_{\infty-(i+2 j-2 p)} D_{\infty-p} .
$$

Furthermore, the maps $\phi_{k}$ induce a map

$$
\phi_{\infty}: \mathcal{A} \rightarrow \mathcal{K}(\infty)
$$

that is an isomorphism of vector spaces and preserves topological degrees (which are both negative).

Proof. Since we defined $\mathcal{K}(\infty)$ as the bigraded limit of the $\mathcal{K}(k)$, we see that for every bidegree $(n, i), \mathcal{K}(\infty)_{n, i}$ is isomorphic to $\mathcal{K}(k)_{n,\left(2^{n}-1\right) k-i}$ for $k$ sufficiently large.

Let $D_{\infty-i}$ be defined by the sequence $\left\{D_{k-i} \mid D_{k-i} \in \mathcal{K}(k)\right\}$. The relations will follow from taking representatives $D_{k-i}$ and $D_{k-j}$ in $\mathcal{K}(k)$ for $k>\max (i, j)$. Then if $i<j$, we have $k-i>k-j$, so

$$
D_{k-i} D_{k-j}=\sum\left(\begin{array}{c}
q-1-(k-j) \\
2 q-(k-i)-(k-j)
\end{array}\right) D_{(k-i)+2(k-j)-2 q} D_{q} .
$$

Setting $p=k-q$, we get

$$
D_{k-i} D_{k-j}=\sum\left(\begin{array}{c}
j-p-1 \\
i+j-2 p
\end{array}\right) D_{k-i-2 j+2 p} D_{k-p},
$$

as desired. 
Since $\bigcap \operatorname{ker}\left(\phi_{k}\right)=\{0\}$, it follows that $\phi_{\infty}$ is an isomorphism.

Remark 6.6. Given how we created $\mathcal{K}(\infty)$, it should be no surprise that the relations of Theorem 6.5 have a formal power series representation related to that of $\mathcal{K}$. Indeed we have

$$
D_{\infty}\left(u^{-1}\right) D_{\infty}\left((u+v)^{-1} v^{-1}\right)=D_{\infty}\left(v^{-1}\right) D_{\infty}\left((u+v)^{-1} u^{-1}\right)
$$

(where $D_{\infty}(x)$ denotes $\sum D_{\infty-i} x^{i}$ ), which may be verified by the residue method of section 3 .

Remark 6.7. $\phi_{\infty}$ is given on admissibles by the formula

$$
\phi_{\infty}\left(S q^{i_{1}} \cdots S q^{i_{n}}\right)=D_{\infty-\left(i_{1}-\left(i_{2}+\cdots+i_{n}\right)\right)} \cdots D_{\infty-\left(i_{n-1}-i_{n}\right)} D_{\infty-i_{n}} .
$$

As usual, $\mathcal{K}(\infty)$ has a vector space basis of admissibles, where a monomial $D_{\infty-i_{1}} \cdots$ $D_{\infty-i_{n}}$ is admissible provided that $i_{k} \geq i_{k+1}$ for all $k$, and we see that $\phi_{\infty}$ is a bijection between both sets of admissibles.

We shall relate the product in $\mathcal{A}$ to the product in $\mathcal{K}(\infty)$ by showing that the inverse of $\phi_{\infty}$ is a sheared algebra bijection. The map $\phi_{\infty}$ itself fails because of the presence of unstable Adem relations.

Definition 6.8. Let the inverse of $\phi_{\infty}: \mathcal{A} \rightarrow \mathcal{K}(\infty)$ be denoted by

$$
\psi_{\infty}: \mathcal{K}(\infty)_{*, i} \rightarrow \mathcal{A}^{i}
$$

A short calculation based on Remark 6.7 yields the formula for $\psi_{\infty}$ on admissibles:

\section{Lemma 6.9.}

$$
\begin{aligned}
& \psi_{\infty}\left(D_{\infty-i_{1}} \cdots D_{\infty-i_{n}}\right) \\
& \quad=S q^{i_{1}+i_{2}+2 i_{3}+\cdots+2^{n-2} i_{n}} \cdots S q^{i_{n-2}+i_{n-1}+2 i_{n}} S q^{i_{n-1}+i_{n}} S q^{i_{n}} .
\end{aligned}
$$

Note that $\psi_{\infty}$ already has the substantial virtue that the RHS of the above formula at least makes sense for any input (not necessarily admissible) on the LHS, while this failed for the $\phi_{\infty}$ formula.

Remark 6.10. While we could have defined $\psi_{\infty}$ directly without first producing $\phi_{\infty}$ via the $\phi_{k}$, we would not be able to have $\psi_{\infty}$ arise directly from maps $\psi_{k}$ out of the inverse limit, since maps out of an inverse limit are not generally forthcoming.

The map $\alpha$ induces an algebra endomorphism

$$
\alpha_{\infty}: \mathcal{K}(\infty)_{n,-i} \rightarrow \mathcal{K}(\infty)_{n,-i-\left(2^{n}-1\right)},
$$

with $\alpha_{\infty}\left(D_{\infty-i}\right)=D_{\infty-(i+1)}$, which we will use in our analogue of Theorem 5.17 Theorem $\mathrm{H}$ follows from

Theorem 6.11. The map $\psi_{\infty}: \mathcal{K}(\infty) \rightarrow \mathcal{A}$ is a sheared algebra bijection, given on arbitrary products by the formula of Lemma 6.9

Proof. To apply Theorem [E] we first note that $\mathcal{K}(\infty)$ is clearly a $\mathrm{P}-\mathrm{B}-\mathrm{W}$ algebra. It is also an algebra with shifting, taking $d$ to be the negative of topological degree and $b=2$, and $F=\alpha_{\infty}$ with $a=1$, since $\alpha_{\infty}$ is an algebra map. Assumption [3 is verified by

$$
d\left(\alpha_{\infty}\left(D_{\infty-i}\right)\right)=d\left(D_{\infty-(i+1)}\right)=i+1=d\left(D_{\infty-i}\right)+b-a .
$$


We then apply Theorem $\mathrm{F}$ by first defining $\psi_{\infty}\left(D_{\infty-i}\right)=S q^{i}$, and verifying the hypothesis that Adem relations are respected, which is again a straightforward calculation.

\section{The Relationship Between $\mathcal{K}^{*}$ And $\mathcal{A}^{*}$}

The relationship in Remark 5.18 between the coproduct formulas in $\mathcal{K}^{*}$ and $\mathcal{R}^{*}$ arose since $\theta_{0}: \mathcal{R} \rightarrow \mathcal{K}$ was a sheared algebra bijection. We intend to shed an analogous light on why the coproduct formula in $\mathcal{A}^{*}$ resembles that of $\mathcal{K}^{*}$ with $x_{i, j}$ replaced by $\xi_{i}$, a most curious relationship since this replacement puzzlingly converts the nonunits $x_{0, n}$ into the unit $\xi_{0}=1$, and converts each unit $x_{n, 0}$ into a nonunit $\xi_{n}$. We shall show how to make sense of this conundrum. It will be elucidated by the connection between the coproduct formulas in $\mathcal{A}^{*}$ and $\mathcal{K}^{*}$ given by the map $\psi_{\infty}^{*}: \mathcal{A}^{*} \rightarrow \mathcal{K}(\infty)^{*}$ dual to the sheared algebra bijection $\psi_{\infty}: \mathcal{K}(\infty) \rightarrow \mathcal{A}$. As with $\mathcal{K}^{*}, \mathcal{K}(\infty)^{*}$ is defined to be the bigraded dual to $\mathcal{K}(\infty)$, and we shall call the dual bidegrees the "length" and "topological" degrees. Thus $\mathcal{K}(\infty)_{n, q}^{*}=$ $\left(\mathcal{K}(\infty)_{n,-q}\right)^{*}$. (We note that $\mathcal{K}(\infty)^{*}$ appears in positive topological degrees.) We shall first describe a standard basis for $\mathcal{K}(\infty)^{*}$, then interpret $\psi_{\infty}^{*}$ in terms of this basis and the standard monomial basis of $\mathcal{A}^{*}$.

We begin by finding appropriate bases for the bialgebras $\mathcal{K}(k)^{*}$.

Proposition 7.1. A basis for $\mathcal{K}(k)_{n, *}^{*}$ is given by the images under the projection $\mathcal{K}^{*} \rightarrow \mathcal{K}(k)^{*}$ of all monomials in $x_{0, n}, \ldots, x_{n-1,1}$ of total exponent $\leq k-1$.

Proof. We dualize the exact sequence of Proposition 6.2 and obtain

$$
0 \rightarrow \mathcal{K}(k-1)_{n, *}^{*} \stackrel{\alpha^{*}}{\rightarrow} \mathcal{K}(k)_{n, *}^{*} \stackrel{\kappa^{*}}{\rightarrow} \mathcal{K}(k)_{n-1, *}^{*} \rightarrow 0 .
$$

The proposition follows from an induction on $k+n$ based on the facts that $\mathcal{K}(k)_{0, *}^{*}$ has the single non-zero element $x_{0,0}$, and that $\mathcal{K}(0)=0$. To see that the elements of the specified form are independent, we form in $\mathcal{K}_{n, *}^{*}$ a sum $x=\sum_{j} X_{j}+\sum_{i} x_{0, n} Y_{i}$ of distinct monomials, where the $X_{j}$ are of exponent $\leq k-1$ and do not have $x_{0, n}$ as a factor, and the $Y_{i}$ are of exponent $\leq k-2$. We ask whether or not $x$ projects to zero in $\mathcal{K}(k)_{n, *}^{*}$. By Example 3.18, $\kappa^{*}(x)=\sum_{j} X_{j}^{\prime}$, where $X_{j}^{\prime}$ is $X_{j}$ with the first indices of all factors reduced by one, and we know inductively that these projections are distinct basis elements of $\mathcal{K}(k)_{n-1, *}^{*}$. So if $x$ projects to zero, all of its summands must have $x_{0, n}$ as a factor. In this case, Example 3.18 shows that $x=\alpha^{*}\left(\sum_{i} Y_{i}\right)$. Again, we know inductively that $\sum_{i} Y_{i}$ projects nonzero to $\mathcal{K}(k-1)_{n, *}^{*}$. Since $\alpha^{*}$ is monomorphic on $\mathcal{K}(k-1)^{*}$, we see that if $x$ projects to zero, all the $Y_{i}$ must also be zero and hence so is $x$. Thus the images of these monomials are independent. Inductively, the $X_{j}^{\prime}$ and $Y_{i}$ form bases for the ends of the exact sequence, and thus the $X_{j}$ and $x_{0, n} Y_{i}$ are a basis for $\mathcal{K}(k)_{n, *}^{*}$, hence the proposition.

Remark 7.2. The algebra epimorphism $\mathcal{K}^{*} \rightarrow \mathcal{K}(k)^{*}$ has been studied by [CPS], in which they prove Proposition 7.1. They also describe an algorithm to resolve the rather complicated problem of determining the images of monomials with higher exponent. While, in general, these images can be complicated, it follows from Corollary 3.12 that $x_{i, j}^{2^{a}}=\left(D_{0}^{i} D_{2^{a}}^{j}\right)^{*}$, so $x_{i, j}^{2^{a}}=0$ in $\mathcal{K}(k)^{*}$ if $2^{a} \geq k$.

We again recall from Example 3.18 that $\alpha^{*}: \mathcal{K}(k)_{n, *}^{*} \rightarrow \mathcal{K}(k+1)_{n, *}^{*}$ is given by multiplication by $x_{0, n}$, which is monomorphic. As a consequence, $\mathcal{K}(\infty)^{*}$ can be identified as the union of the $\mathcal{K}(k)^{*}$ under the inclusions $\alpha^{*}$. Thus a monomial 
$x_{0, n}^{l_{0}} x_{1, n-1}^{l_{1}} \cdots x_{n-1,1}^{l_{n-1}}$ in $\mathcal{K}(k)^{*}$ is identified with the monomial $x_{0, n}^{l_{0}+1} x_{1, n-1}^{l_{1}} \cdots x_{n-1,1}^{l_{n-1}}$ in $\mathcal{K}(k+1)^{*}$, and hence we obtain the following result, which includes Proposition I.

Proposition 7.3. A basis for $\mathcal{K}(\infty)^{*}$ is given by "monomials"

$$
x_{0, n}^{-l} x_{1, n-1}^{l_{1}} \cdots x_{n-1,1}^{l_{n-1}} \in \mathcal{K}(\infty)_{n, t}^{*} \quad \text { for } l>\sum_{i=1}^{n-1} l_{i},
$$

where $t=l\left(2^{n}-1\right)-\sum l_{i} 2^{i}\left(2^{n-i}-1\right)$. The "monomial" above represents the elements $x_{0, n}^{k-l} x_{1, n-1}^{l_{1}} \cdots x_{n-1,1}^{l_{n-1}}$ in $\mathcal{K}(k)^{*}$ for $k \geq l$.

As mentioned in section 2 we do not know how to compute the coproducts of all elements of $\mathcal{K}(\infty)^{*}$, due to the challenge mentioned above involved in identifying the images of elements under the projections $\mathcal{K}^{*} \rightarrow \mathcal{K}(k)^{*}$. But we can compute certain ones. For instance, if $n \geq m$, then using the formula for the coproduct in $\mathcal{K}^{*}$ and Remark 7.2 .

$$
\mu_{\mathcal{K}(p)}^{*}\left(x_{m, n-m}\right)=\sum_{\substack{0 \leq k \leq n-m \\ \max \left\{1, m-\log _{2} p\right\}<j \leq m}} x_{j, k}^{2^{m-j}} \otimes x_{m-j,(n-m)-k}
$$

is a formula for the coproduct given in the chosen basis elements for $\mathcal{K}(p)^{*}$. Hence in $\mathcal{K}(\infty)^{*}$, we have

Lemma 7.4. (1) If $E \geq 1$, then

$$
\mu_{\infty}^{*}\left(x_{0, n}^{-E} x_{m, n-m}\right)=\sum_{\substack{0 \leq k \leq n-m \\ 0 \leq j \leq m}} x_{0, j+k}^{-E} x_{j, k}^{2^{m-j}} \otimes x_{0, n-j-k}^{-E} x_{m-j,(n-m)-k}
$$

(in which the previously undefined symbols $x_{0, j+k}^{-E} x_{j, k}^{2^{m-j}}$ for $E \leq 2^{m-j}$ are taken to be zero), and

(2) if $l>0$, then

$$
\mu_{\infty}^{*}\left(x_{0, n}^{-l}\right)=\sum x_{0, k}^{-l} \otimes x_{0, n-k}^{-l}
$$

Before proving our "leading term" theorem, Theorem D, we will first see how it sheds light on the relationship between the coproducts in $\mathcal{K}^{*}$ and $\mathcal{A}^{*}$.

We begin by noting, using Example 3.18, that the dual of $\alpha_{\infty}: \mathcal{K}(\infty) \rightarrow \mathcal{K}(\infty)$,

$$
\alpha_{\infty}^{*}: \mathcal{K}(\infty)_{n, i}^{*} \rightarrow \mathcal{K}(\infty)_{n, i+\left(2^{n}-1\right)}^{*}
$$

takes the form $\alpha_{\infty}^{*}\left(x_{0, n}^{-l} x\right)=x_{0, n}^{-l+1} x$. From Remark 2.5] the shearing property of $\psi_{\infty}$ in Theorem 6.11 may be written as

$$
\psi_{\infty} \circ \mu_{\infty}=\mu_{\mathcal{A}} \circ\left(\psi_{\infty} \otimes \psi_{\infty}\right) \circ \gamma
$$

where

$$
\gamma\left(z \otimes z^{\prime}\right)=F^{d\left(z^{\prime}\right)}(z) \otimes z^{\prime}=\left(\alpha_{\infty}\right)^{-\left|z^{\prime}\right|}(z) \otimes z^{\prime}
$$

and $\left|z^{\prime}\right|$ is the (negative) topological degree of $z^{\prime} \in \mathcal{K}(\infty)_{*, *}$. We dualize to obtain

$$
\gamma^{*} \circ\left(\psi_{\infty}^{*} \otimes \psi_{\infty}^{*}\right) \circ \mu_{\mathcal{A}}^{*}=\mu_{\infty}^{*} \circ \psi_{\infty}^{*},
$$

where $\gamma^{*}$ is given by $\left(\alpha_{\infty}^{*}\right)^{t} \otimes 1$ on $\mathcal{K}(\infty)^{*} \otimes \mathcal{K}(\infty)_{*, t}^{*}$. We first see what is revealed by applying this equality to $\xi_{n}$. All calculations in $\mathcal{K}(\infty)^{*}$ will be modulo length degree $>n$. 
We start with

$$
\psi_{\infty}^{*}\left(\xi_{n}\right)=x_{0, n}^{-1},
$$

from Theorem J. Then by Lemma 7.4 we have

$$
\mu_{\infty}^{*} \psi_{\infty}^{*}\left(\xi_{n}\right)=\sum_{j=0}^{n} x_{0, j}^{-1} \otimes x_{0, n-j}^{-1}
$$

for the right side of the equality.

On the left side,

$$
\mu_{\mathcal{A}}^{*}\left(\xi_{n}\right)=\sum_{j=0}^{n} \xi_{j}^{2^{n-j}} \otimes \xi_{n-j},
$$

hence

$$
\left(\psi_{\infty}^{*} \otimes \psi_{\infty}^{*}\right) \mu_{\mathcal{A}}^{*}\left(\xi_{n}\right)=\sum_{j=0}^{n} x_{0, j}^{-2^{n-j}} \otimes x_{0, n-j}^{-1} .
$$

Now $x_{0, n-j}^{-1} \in \mathcal{K}(\infty)_{n-j, 2^{n-j}-1}^{*}$, so

$$
\begin{gathered}
\gamma^{*}\left(\psi_{\infty}^{*} \otimes \psi_{\infty}^{*}\right) \mu_{\mathcal{A}}^{*}\left(\xi_{n}\right)=\sum_{j=0}^{n}\left(\alpha_{\infty}^{*}\right)^{2^{n-j}-1}\left(x_{0, j}^{-2^{n-j}}\right) \otimes x_{0, n-j}^{-1} \\
=\sum_{j=0}^{n} x_{0, j}^{-1} \otimes x_{0, n-j}^{-1},
\end{gathered}
$$

for the left side. So we see first that the coproduct formula on $\xi_{n}$ is essentially forced by the apparently simpler coproduct formula on $x_{0, n}$, together with the co-shearing feature of the map $\psi_{\infty}^{*}$.

Next, we shall turn the tables around to see how, in the other direction, the coproducts of the $x_{i, j}$ are essentially forced by the coproducts of the $\xi_{n}$ and the co-shearing of $\psi_{\infty}^{*}$. Let $E=2^{m}$ and suppose that $n \geq m$. Using Theorem J and Lemma 7.4, we compute that

$$
\begin{aligned}
\mu_{\infty}^{*} \psi_{\infty}^{*}\left(\xi_{n}^{E} \xi_{m}\right)=\mu_{\infty}^{*}\left(x_{0, n}^{-(E+1)} x_{m, n-m}\right) & =\sum_{\substack{0 \leq k \leq n-m \\
0 \leq j \leq m}} x_{0, j+k}^{-(E+1)} x_{j, k}^{2^{m-j}} \otimes x_{0, n-j-k}^{-(E+1)} x_{m-j,(n-m)-k} .
\end{aligned}
$$

On the other hand,

$$
\mu_{\mathcal{A}}^{*}\left(\xi_{n}^{E} \xi_{m}\right)=\sum_{\substack{0 \leq i \leq n \\ 0 \leq j \leq m}} \xi_{i}^{2^{n-i} E} \xi_{j}^{2^{m-j}} \otimes \xi_{n-i}^{E} \xi_{m-j} .
$$

The pairs of indices break into two cases: (1) $i \geq j$ and $n-i \geq m-j$; and (2) otherwise. In case (2), the image of this under $\psi_{\infty}^{*} \otimes \psi_{\infty}^{*}$ lands in total length degree $>n$. So modulo this filtration we get, again from Theorem Џ,

$$
\begin{aligned}
\left(\psi_{\infty}^{*}\right. & \left.\otimes \psi_{\infty}^{*}\right) \mu_{\mathcal{A}}^{*}\left(\xi_{n}^{E} \xi_{m}\right) \\
& =\sum_{\substack{0 \leq j \leq m \\
0 \leq i-j \leq n-m}} x_{0, i}^{-\left(2^{n-i} E+2^{m-j}\right)} x_{j, i-j}^{2^{m-j}} \otimes x_{0, n-i}^{-(E+1)} x_{m-j,(n-i)-(m-j)} .
\end{aligned}
$$


The topological degree of the right-hand factor, $x_{0, n-i}^{-(E+1)} x_{m-j,(n-i)-(m-j)}$, is $(E+1)\left(2^{n-i}-1\right)-2^{m-j}\left(2^{(n-i)-(m-j)}-1\right)$, which simplifies to $2^{n-i} E+2^{m-j}-$ $(E+1)$. So

$$
\begin{aligned}
\gamma^{*}\left(\psi_{\infty}^{*} \otimes \psi_{\infty}^{*}\right) \mu_{\mathcal{A}}^{*}\left(\xi_{n}^{E} \xi_{m}\right) & \\
\quad= & \sum_{\substack{0 \leq j \leq m \\
0 \leq i-j \leq n-m}} x_{0, i}^{-(E+1)} x_{j, i-j}^{2^{m-j}} \otimes x_{0, n-i}^{-(E+1)} x_{m-j,(n-i)-(m-j)}
\end{aligned}
$$

which, letting $k=i-j$, matches our formula above for $\mu_{\infty}^{*} \psi_{\infty}^{*}\left(\xi_{n}^{E} \xi_{m}\right)$. Thus we see the coproduct formula on $x_{m, n-m}$ emerge as essentially forced by the coproduct formulae on $\xi_{n}$ and $\xi_{m}$.

For us these sample calculations of mutual forcing via the cosheared feature of $\psi_{\infty}^{*}$ provide a satisfying answer to the earlier puzzle of how the coproduct formulae in $\mathcal{K}^{*}$ and $\mathcal{A}^{*}$ seem so similar in some ways, and yet in other ways so different.

We shall prove Theorem J using some related maps $\psi_{k}^{*}: \mathcal{A}^{*} \rightarrow \mathcal{K}^{*}$ which we now describe.

First, fix a value for $n$. Let $I=\left(i_{1}, \ldots, i_{n}\right)$ be an $n$-tuple of integers, and $D_{I}=D_{i_{1}} \cdots D_{i_{n}}$.

Definition 7.5. Let $\psi: \mathbb{Z}^{n+1} \rightarrow \mathbb{Z}^{n}$ be given by

$$
\psi(k, I)=\left(2^{n-1} k-2^{n-2} i_{n}-\cdots-2 i_{3}-i_{2}-i_{1}, \ldots, 2 k-i_{n}-i_{n-1}, k-i_{n}\right) .
$$

Note that $\psi$ is linear in the $(n+1)$-tuple $\left(k, i_{1}, \ldots, i_{n}\right)$, and each $\psi\left(k,{ }_{-}\right)$is an isomorphism. We define linear transformations $\psi_{k}: \mathcal{K} \rightarrow \mathcal{A}$ for $k \geq 0$ by

Definition 7.6. If $D_{I}$ is admissible, we set $\psi_{k}\left(D_{I}\right)$ equal to the monomial string $S q^{\psi(k, I)}$, which represents zero in $\mathcal{A}$ if any indices are negative, i.e. if $i_{n}>k$. (We will often need to focus on the specific monomial string $S q^{\psi(k, I)}$, not merely the element of $\mathcal{A}$ which it represents.)

Remark 7.7. The formula for $\psi$ shows that $\psi_{k}\left(D_{I}\right)$ represents an admissible in $\mathcal{A}$ provided $i_{n}<k$, and that if $i_{n}=k$, then it is an admissible string with some trailing $S q^{0}$ 's appended.

Remark 7.8. We note that this definition gives the correspondence between $\mathcal{K}$ and $\mathcal{A}$ acting on a cohomology class of degree $-k$. As such, if $D_{I}$ is an arbitrary (not necessarily admissible) monomial, then we can see, from a universal example such as $K(\mathbb{Z} / 2, k)$, that it follows that $\psi_{k}\left(D_{I}\right)$ is given by the same recipe, provided it is then rewritten in terms of admissibles and those terms with excess greater than $k$ dropped.

We now prepare to prove a leading term theorem for $\psi_{k}^{*}: \mathcal{A}^{*} \rightarrow \mathcal{K}^{*}$, from which the proof of Theorem Will follow.

Definition 7.9. If $S q^{J}=S q^{j_{1}} \cdots S q^{j_{n}}$ is admissible except possibly for some trailing $S q^{0}$ 's, $l \geq 0$, and $0 \leq j \leq n$, set

$$
V^{j}\left(S q^{J}\right)=\sum\left\{S q^{J-\sigma} \mid \sigma \text { is a }(j, n-j) \text {-shuffle of }\left(2^{j-1}, 2^{j-2}, \ldots, 1,0, \ldots, 0\right)\right\},
$$

and let $V_{l}^{j}\left(S q^{J}\right)$ denote $V^{j}\left(S q^{J}\right)$, expressed in terms of admissibles, deleting all summands of length less than $n$ and all those of excess greater than $l-1$. 
Definition 7.10. For $D_{I}$ admissible, define

$$
W^{j}\left(D_{I}\right)=\sum\left\{D_{I-\psi_{1}^{-1}(\sigma)} \mid \sigma \text { is a }(j, n-j) \text {-shuffle of }\left(2^{j-1}, 2^{j-2}, \ldots, 1,0, \ldots, 0\right)\right\} \text {. }
$$

Lemma 7.11. If $D_{I}$ is admissible, then modulo the subspace of $\mathcal{A}$ with basis the set of admissibles of length less than $n$,

$$
V_{k}^{j}\left(\psi_{k}\left(D_{I}\right)\right)=\psi_{k-1}\left(W^{j}\left(D_{I}\right)\right) \text {. }
$$

Proof. Let

$$
X=\left\{\sigma \mid \sigma \text { is a }(j, n-j) \text {-shuffle of }\left(2^{j-1}, 2^{j-2}, \ldots, 1,0, \ldots, 0\right)\right\} .
$$

Then modulo the stated subspace of $\mathcal{A}$, and using Definitions 7.6 and 7.10, Remark 7.8, and the linearity of $\psi$,

$$
\begin{gathered}
\psi_{k-1}\left(W^{j}\left(D_{I}\right)\right)=\sum\left\{\psi_{k-1}\left(D_{I-\psi_{1}^{-1}(\sigma)}\right) \mid \sigma \in X\right\} \\
=\sum\left\{S q^{\psi\left(k-1, I-\psi_{1}^{-1}(\sigma)\right)} \mid \sigma \in X\right\}
\end{gathered}
$$

(with summands of excess $>k-1$ dropped after conversion to admissibles)

$$
=\sum\left\{S q^{\psi(k, I)-\sigma} \mid \sigma \in X\right\}
$$

(with summands of excess $>k-1$ dropped after conversion to admissibles).

This in turn is by Definition 7.9 and Remark 7.7 equal to $V_{k}^{j}\left(S q^{\psi(k, I)}\right)$ if $\psi(k, I)$ is nonnegative, and also otherwise, since both will then represent zero. Finally, $V_{k}^{j}\left(S q^{\psi(k, I)}\right)=V_{k}^{j}\left(\psi_{k}\left(D_{I}\right)\right)$.

Theorem Ð will follow from

Theorem 7.12. Let $l=\sum_{i=1}^{n} l_{i}$. If $l_{n} \geq 1$ and $k \geq l$, then modulo $\bigoplus_{m>n} \mathcal{K}_{m, *}^{*}$,

$$
\psi_{k}^{*}\left(\xi_{1}^{l_{1}} \ldots \xi_{n}^{l_{n}}\right)=x_{0, n}^{k-l} x_{1, n-1}^{l_{1}} \cdots x_{n-1,1}^{l_{n-1}} .
$$

Our proof of this will rely first on a special case in slightly stronger form.

Lemma 7.13. Let $l \geq 1$. Modulo $\bigoplus_{m>n} \mathcal{K}_{m, *}^{*}$,

$$
\psi_{k}^{*}\left(\xi_{n}^{l}\right)= \begin{cases}x_{0, n}^{k-l} & \text { if } k \geq l, \\ 0 & \text { otherwise. }\end{cases}
$$

Proof. Let $D_{I}$ be admissible of length at most $n$. We evaluate

$$
\begin{aligned}
& \left\langle\psi_{k}^{*}\left(\xi_{n}^{l}\right), D_{I}\right\rangle=\left\langle\xi_{n}^{l}, \psi_{k}\left(D_{I}\right)\right\rangle=\left\langle\xi_{n} \otimes \cdots \otimes \xi_{n}, \Delta^{l-1}\left(\psi_{k}\left(D_{I}\right)\right)\right\rangle \\
& \quad=\left\{\begin{array}{cl}
1 & \text { if } \Delta^{l-1}\left(\psi_{k}\left(D_{I}\right)\right) \text { contains the term } \\
\left(S q^{2^{n-1}} \cdots S q^{2} S q^{1}\right)^{\otimes l} \text { when expressed using admissibles, } & \text { otherwise. }
\end{array}\right.
\end{aligned}
$$

But the only admissible $S q^{J}$ of length $\leq n$ for which $\Delta^{l-1}\left(S q^{J}\right)$ contains this term is $S q^{2^{n-1} l} \cdots S q^{l}$, and since $\psi_{k}\left(D_{I}\right)$ contains this term in its admissible representation if and only if $D_{I}=D_{k-l}^{n}$, we have $\psi_{k}^{*}\left(\xi_{n}^{l}\right)=\left(D_{k-l}^{n}\right)^{*}$ modulo length $n$. By Proposition 3.20, $x_{0, n}^{k-l}=\left(D_{k-l}^{n}\right)^{*}$ in $\mathcal{K}^{*}$, whence the lemma.

Proof of Theorem 7.12. We shall freely use the following facts: If $w=S q^{i_{1}} \cdots S q^{i_{m}}$ is admissible with $m<n$, then $w$ pairs to 0 with the ideal generated by $\xi_{n}, \xi_{n+1}, \ldots$. If the excess of an admissible $S q^{J}$ is greater than $q$, then $S q^{J}$ pairs trivially with any monomial in the $\xi$ 's of total exponent $q$ or less. 


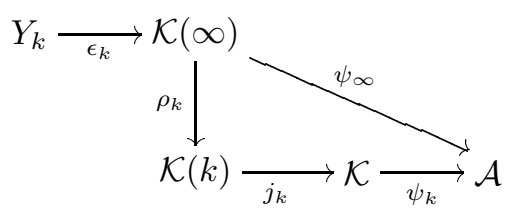

FiguRE 2 .

Inductively, based on Lemma 7.13 , which includes the case $l=1$, assume that the desired formula holds for total exponents less than $l$. Let $j<n$ be the smallest $j$ for which $l_{j}>0$ (the case where this smallest $j$ equals $n$ is already covered by Lemma 7.13). Write $\xi=\xi_{j}^{l_{j}-1} \xi_{j+1}^{l_{j+1}} \ldots \xi_{n-1}^{l_{n-1}}$ and let $x \in \mathcal{K}$ be defined correspondingly. Let $D_{I}$ be admissible of length $\leq n$. If the length of $D_{I}$ is strictly less than $n$, then

$$
\left\langle\psi_{k}^{*}\left(\xi_{j} \xi \xi_{n}^{l_{n}}\right), D_{I}\right\rangle=0=\left\langle x_{j, n-j} x x_{0, n}^{k-l}, D_{I}\right\rangle,
$$

since $\psi_{k}\left(D_{I}\right)$ also has length $<n$.

So assume that $D_{I}$ is admissible of length $n$. We compute

$$
\begin{aligned}
& \left\langle\psi_{k}^{*}\left(\xi_{j} \xi \xi_{n}^{l_{n}}\right), D_{I}\right\rangle=\left\langle\xi_{j} \xi \xi_{n}^{l_{n}}, \psi_{k}\left(D_{I}\right)\right\rangle \\
& \quad=\left\langle\xi_{j} \otimes \xi \xi_{n}^{l_{n}}, \Delta\left(\psi_{k}\left(D_{I}\right)\right)\right\rangle \\
& =\left\langle\xi \xi_{n}^{l_{n}}, V^{j}\left(\psi_{k}\left(D_{I}\right)\right)\right\rangle \text { by Remark 7.7 and Definition } 7.9 \\
& =\left\langle\xi \xi_{n}^{l_{n}}, V_{l}^{j}\left(\psi_{k}\left(D_{I}\right)\right)\right\rangle \text { by Definition } 7.9 \\
& =\left\langle\xi \xi_{n}^{l_{n}}, V_{k}^{j}\left(\psi_{k}\left(D_{I}\right)\right)\right\rangle \text { since } k \geq l \\
& =\left\langle\xi \xi_{n}^{l_{n}}, \psi_{k-1}\left(W^{j}\left(D_{I}\right)\right)\right\rangle \text { from Lemma } 7.11 \\
& =\left\langle\psi_{k-1}^{*}\left(\xi \xi_{n}^{l_{n}}\right), W^{j}\left(D_{I}\right)\right\rangle \\
& =\left\langle x x_{0, n}^{k-l}, W^{j}\left(D_{I}\right)\right\rangle \quad \text { by induction, since } k-1 \geq l-1, \text { and } \\
& \quad \text { since } W^{j}\left(D_{I}\right) \text { has length } \leq n .
\end{aligned}
$$

Finally, $\left\langle x x_{0, n}^{k-l}, W^{j}\left(D_{I}\right)\right\rangle=\left\langle x_{j, n-j} x x_{0, n}^{k-l}, D_{I}\right\rangle$ from Definition 7.10 since using Lemma 3.7 one can check that the elements $\psi_{1}^{-1}(\sigma)$ are precisely the length $n$ monomials which involve the admissible basis element $D_{0}^{j} D_{1}^{n-j}$ (in fact, they each equal it).

Proof of Theorem Џ. Choose $k$ to be at least the topological degree of $\xi_{1}^{l_{1}} \ldots \xi_{n}^{l_{n}}$. Let $Y_{k}$ denote the subspace of $\mathcal{K}(\infty)$ with basis the set of admissibles

$$
\left\{D_{\infty-I}=D_{\infty-i_{1}} \cdots D_{\infty-i_{q}} \mid q \geq 0 \text { and } i_{1} \leq k\right\} .
$$

We note first that in topological degrees $\geq-k, Y_{k}$ is isomorphic to $\mathcal{K}(\infty)$. Second, let $\rho_{k}: \mathcal{K}(\infty) \rightarrow \mathcal{K}(k)$ denote the projection, and $j_{k}: \mathcal{K}(k) \rightarrow \mathcal{K}$ and $\epsilon_{k}: Y_{k} \rightarrow$ $\mathcal{K}(\infty)$ the inclusions. By following the bases we see that $\rho_{k} \circ \epsilon_{k}$ is an isomorphism, and that

$$
\psi_{\infty} \circ \epsilon_{k}=\psi_{k} \circ j_{k} \circ \rho_{k} \circ \epsilon_{k}
$$

by direct calculation using our formulas on admissibles for $\psi_{k}$ and $\psi_{\infty}$ (even though we caution that $\left.\psi_{\infty} \neq \psi_{k} \circ j_{k} \circ \rho_{k}\right)$. We summarize this in Figure 2 in which the triangle is not commutative until composed with $\epsilon_{k}$. 
Therefore

$$
\epsilon_{k}^{*} \circ \psi_{\infty}^{*}\left(\xi_{1}^{l_{1}} \cdots \xi_{n}^{l_{n}}\right)=\epsilon_{k}^{*} \circ \rho_{k}^{*} \circ j_{k}^{*} \circ \psi_{k}^{*}\left(\xi_{1}^{l_{1}} \cdots \xi_{n}^{l_{n}}\right) .
$$

Now $\psi_{k}^{*}\left(\xi_{1}^{l_{1}} \cdots \xi_{n}^{l_{n}}\right)=x_{0, n}^{k-l} x_{1, n-1}^{l_{1}} \cdots x_{n-1,1}^{l_{n-1}}$ modulo $\bigoplus_{m>n} \mathcal{K}_{m, *}^{*}$ from Theorem 7.12 since $k \geq \operatorname{deg}\left(\xi_{1}^{l_{1}} \ldots \xi_{n}^{l_{n}}\right) \geq l$. Thus, since $\epsilon_{k}, \rho_{k}, j_{k}$ are each length-preserving, we have

$$
\begin{aligned}
& \epsilon_{k}^{*} \circ \rho_{k}^{*} \circ j_{k}^{*} \circ \psi_{k}^{*}\left(\xi_{1}^{l_{1}} \cdots \xi_{n}^{l_{n}}\right) \\
& \quad=\epsilon_{k}^{*} \circ \rho_{k}^{*} \circ j_{k}^{*}\left(x_{0, n}^{k-l} x_{1, n-1}^{l_{1}} \cdots x_{n-1,1}^{l_{n-1}}\right) \text { modulo } \bigoplus_{m>n} \mathcal{K}(\infty)_{m, *}^{*},
\end{aligned}
$$

which in turn equals

$$
\epsilon_{k}^{*}\left(x_{0, n}^{-l} x_{1, n-1}^{l_{1}} \cdots x_{n-1,1}^{l_{n-1}}\right)
$$

since the exponent of $x_{0, n}^{k-l} x_{1, n-1}^{l_{1}} \cdots x_{n-1,1}^{l_{n-1}}$ is less than $k$. Now since $\epsilon_{k}^{*}$ is an isomorphism in this degree, the result follows.

\section{The Cohomology of $\mathcal{K}$}

In this section we shall use methods of $[\mathrm{Pr}]$ to compute the cohomology $\operatorname{Ext}_{\mathcal{K}}\left(\mathbf{F}_{2}, \mathbf{F}_{2}\right)$ of $\mathcal{K}$. (We suggest [Lo] as a concise reference for the cohomology of P-B-W algebras.) We shall then construct a sheared algebra bijection to it from the algebra $\mathcal{K}(\infty)$ of section 6 . Finally, we shall construct a sheared algebra bijection from it to $\mathcal{A}$, consider the effect of sheared algebra maps on homology and cohomology, summarize how our sheared bijections fit together, and suggest applications via the Miller spectral sequence.

We begin by recalling the defining relations of $\mathcal{K}$ from Definition 2.1

$$
D_{i} D_{j}=\sum_{k}\left(\begin{array}{c}
k-1-j \\
2 k-i-j
\end{array}\right) D_{i+2 j-2 k} D_{k}, \quad(i>j) .
$$

Following $[\mathrm{Pr}$, we define

$$
m=i+2 j-2 k \text {. }
$$

We note that

$$
\left(\begin{array}{c}
k-1-j \\
2 k-i-j
\end{array}\right)=\left(\begin{array}{c}
k-1-j \\
j-m
\end{array}\right)
$$

It is clear that $\mathcal{K}$ is a $\mathrm{P}-\mathrm{B}-\mathrm{W}$ algebra in our setting, with skew-additive topological degree with $b=2$ in Assumption 1. The next proposition then follows from [Pr]. Before stating it, we shall make a brief remark on gradings:

Remark 8.1. The cohomology of $\mathcal{K}$ is $H^{*}(\mathcal{K})=\operatorname{Ext}_{\mathcal{K}}\left(\mathbf{F}_{2}, \mathbf{F}_{2}\right)$. As is pointed out in $[\mathrm{Pr}$, since a $\mathrm{P}-\mathrm{B}-\mathrm{W}$ algebra is bigraded, the cohomology is trigraded. The elements in $H^{i, n, k}(\mathcal{K})$ are defined by Priddy to be of cohomological degree $i$, length degree $n$, and internal degree $k$. For consistency with our convention of grading cohomology negatively, we define the topological degree of such elements to be $-(i+k)$. 
Proposition 8.2. The cohomology of $\mathcal{K}, H^{*}(\mathcal{K})$, is the algebra with generators $\sigma_{k} \in \operatorname{Ext}^{1,1, k}(k \geq 0)$ and Adem relations, for $m \leq k$,

$$
\sigma_{m} \sigma_{k}=\sum\left(\begin{array}{c}
k-1-j \\
j-m
\end{array}\right) \sigma_{m+2 k-2 j} \sigma_{j} .
$$

The internal degree of products behaves in analogous fashion to that of $\mathcal{K}$, i.e. skewadditively with $b=2$ in Assumption 1 .

As usual, $H^{*}(\mathcal{K})$ has a basis of admissibles, where a monomial $\sigma_{i_{1}} \cdots \sigma_{i_{n}}$ is admissible provided that $i_{l}>i_{l+1}$ for all $l$. The relations for $H^{*}(\mathcal{K})$ are encoded by

$$
u \sigma\left(u^{-1}\right) \sigma\left((u+v)^{-1} v^{-1}\right)=v \sigma\left(v^{-1}\right) \sigma\left((u+v)^{-1} u^{-1}\right),
$$

which may again be verified by a residue calculation.

We next proceed to develop a sheared algebra bijection

$$
\omega: \mathcal{K}(\infty) \rightarrow H^{*}(\mathcal{K})
$$

We recall that $\mathcal{K}(\infty)$ is an algebra with shifting map $\alpha_{\infty}$. Previously we used the absolute value of the topological degree as a degree map, but we now observe that the length degree $n$ can also serve as a degree map, with $b=1$, and $n$ interacts with $\alpha_{\infty}$ (with $a=1$ ) in the desired way (Assumption 3) to again produce an algebra with shifting $\left(\mathcal{K}(\infty),\left\{D_{\infty-i}\right\}, \alpha_{\infty}, n\right)$. In the now-standard way, we define $\omega$ on generators by the formula

$$
\omega\left(D_{\infty-i}\right)=\sigma_{i-1} .
$$

Theorem 8.3. The map $\omega$ is a sheared algebra bijection which preserves topological degree. Thus it is given by

$$
\omega\left(\cdots D_{\infty-c} D_{\infty-b} D_{\infty-a}\right)=\cdots=\sigma_{c+1} \sigma_{b} \sigma_{a-1}
$$

on any monomial, not necessarily admissible.

Proof. A direct calculation confirms that $\omega$ respects Adem relations as required by Theorem E. Alternatively, one may do a pleasant calculation comparing the formal power series forms of the Adem relations. This yields the sheared algebra map and formula. It is then immediate that it preserves topological degree and is a bijection.

We now turn to the relationship between $H^{*}(\mathcal{K})$ and $\mathcal{A}$.

As we observed in Proposition 8.2 the internal degree of products in $H^{*}(\mathcal{K})$ behaves analogously to that in $\mathcal{K}$ and so internal degree will serve as a degree function for $H^{*}(\mathcal{K})$, with constant $b=2$. To obtain a shifting map, we need only define

$$
s\left(\sigma_{i}\right)=\sigma_{i+1},
$$

and verify Assumption 2. This is easily checked, with constant $a=1$, by comparison of binomial coefficients, or by formal power series via the method mentioned for $\alpha$ after Example 3.18, since here $s(\sigma(x))=x^{-1} \sigma(x)$. Finally, $s$ interacts with internal degree $i$ as required by Assumption 3 . We have

Proposition 8.4. The map $s$ is an algebra endomorphism, and we have an algebra with shifting $\left(H^{*}(\mathcal{K}),\left\{\sigma_{i}\right\}, s, i\right)$. 
We intend to apply Theorem $\mathrm{F}$ to obtain yet another sheared algebra bijection, this time from $H^{*}(\mathcal{K})$ to $\mathcal{A}$. But first, note that we could simply compose the isomorphisms $\omega^{-1}: H^{*}(\mathcal{K}) \rightarrow \mathcal{K}(\infty)$ and $\psi_{\infty}: \mathcal{K}(\infty) \rightarrow \mathcal{A}$ to obtain an isomorphism of vector spaces,

$$
\nu: H^{*}(\mathcal{K}) \rightarrow \mathcal{A}
$$

given on admissibles by

$$
\begin{aligned}
\nu\left(\sigma_{i_{1}}\right. & \left.\cdots \sigma_{i_{n}}\right) \\
& =S q^{i_{1}+i_{2}+2 i_{3}+\cdots+2^{n-2} i_{n}+1} \cdots S q^{i_{n-2}+i_{n-1}+2 i_{n}+1} S q^{i_{n-1}+i_{n}+1} S q^{i_{n}+1} .
\end{aligned}
$$

It is immediate that $\nu$ preserves topological degree. It would be nice to claim now that this $\nu$ is a sheared algebra map, i.e. that this formula holds on any monomial, simply by combining Theorems 6.11 and 8.3 , but unfortunately Theorem 8.3 does not yield adequate information about $\omega^{-1}$ (recall that sheared algebra bijections are only one-way affairs). The following theorem, which emerges immediately in the now familiar way using Theorem $[\mathrm{F}$ accomplishes this goal of relating products in $H^{*}(\mathcal{K})$ directly to those in $\mathcal{A}$.

Theorem 8.5. The map $\nu: H^{*}(\mathcal{K}) \rightarrow \mathcal{A}$ is a sheared algebra bijection.

We might equally well consider the Lie Steenrod algebra, $\mathcal{A}_{L}[\mathrm{Lo}, \mathrm{Pr}]$, which is formed by replacing the relation $S q^{0}=1$ with the relation $S q^{0}=0$. Since the image under $\nu$ of any monomial in $H^{*}(\mathcal{K})$ produces a monomial in $\mathcal{A}$ for which the reduction to a sum of admissibles via Adem relations does not produce any terms with factors of $S q^{0}$ (cf. Remark 6.1),$\nu$ can equally well be regarded as a sheared algebra bijection from $H^{*}(\mathcal{K})$ to $\mathcal{A}_{L}$.

Theorems 8.3 and 8.5 give Theorem $[\mathrm{K}$ of section 2 We now turn to the effect of sheared algebra morphisms on homology and cohomology, and note that the proof of Theorem $\mathrm{Q}$ is straightforward using the definition of a sheared algebra homomorphism and Theorems 5.6, 5.11, and 5.14,

Using the formulas of $\overline{\mathrm{Pr}}$, it is easy to see that if $K, M$ are both P-B-W algebras, and if $\psi$ carries generators to generators, one can write a formula for the induced map on cohomology. In particular, one can immediately see that the sheared algebra morphism $\hat{\theta}_{0}: \mathcal{R}(-\infty) \rightarrow \mathcal{K}$ of section 5 induces a vector space isomorphism $H^{*}\left(\hat{\theta}_{0}\right)$ on cohomology that is also a sheared algebra map.

We hope to apply the results of this section to the setting of the Miller spectral sequence [KL1 Mill]. The built-in "unstableness" of the action of $\mathcal{K}$ on the homology of infinite loopspaces should enable the use of the standard Ext functors in place of the somewhat artificial Unext, and thereby result in shortened calculations. Also we feel that, with the simpler coproduct formula of $\mathcal{K}^{*}$ as compared to $\mathcal{R}^{*}$, the range of applicability of the Miller spectral sequence can be extended, leading to more results in the direction of those of [KL2]. We are presently working on such computations.

\section{Future Directions}

As we have seen, the algebra $\mathcal{K}$ has a very rich internal structure. It may be regarded as being closer to the actual topology of spaces than either $\mathcal{A}$ or $\mathcal{R}$, and we hope this, along with the "nice" coproduct formula in $\mathcal{K}^{*}$, may facilitate 
applications. We have also seen how $\mathcal{K}$, and its cohomology, are related to $\mathcal{A}$ and $\mathcal{R}$ via various interrelated sheared algebra bijections.

There are several avenues of further research and application that we are currently pursuing. We briefly list a few.

(1) The work of Bisson-Joyal [BJ] approaches $\mathcal{K}$ from a very different perspective. Combining our point of view with theirs is providing new insights as well as new results. In particular, in [BPW] we use the extended Milnor-Hopf algebra [BJ] to explain the shearing that occurs in our map $\psi_{\infty}: \mathcal{K}(\infty) \rightarrow \mathcal{A}$.

(2) Although the present paper provides a unifying treatment of homology and cohomology operations through the use of $\mathcal{K}$ and its related algebras, it would be nice to see a direct connection between the Steenrod algebra and the Dyer-Lashof algebra. We treat this question in [PW1] in which we develop an inverse limit process, based on the way $\mathcal{K}(\infty)$ was formed from $\mathcal{K}$, to produce the Steenrod algebra from the Dyer-Lashof algebra and vice versa.

(3) As mentioned above, we plan to examine the categorical structure of sheared algebra morphisms in a future paper. We hope to relate this to the concept of gradings over various monoid structures on $\mathbb{Z}^{+} \times \mathbb{Z}^{+}$that was discussed following Definition 2.1

(4) The "Nishida" actions of $\mathcal{K}^{\mathrm{op}}$ on $\mathcal{K}$ based on fundamental classes in positive degrees, mentioned above in section 4 , are closely related to the actions of the Steenrod algebra on the homology and cohomology of iterated loopspaces of spheres. We are interested in describing these actions through use of the maps $\kappa, \alpha$, and $V$ of this paper.

(5) As mentioned in section 8 , we also plan to use homological calculations based on $\mathcal{K}$ to improve and extend the use of the Miller spectral sequence [KL2, Mill in the computation of the homology of infinite loopspaces.

(6) In many cases the statements of theorems become more concise through use of $\mathcal{K}$. For instance, the description of the homology of iterated loop spaces in terms of $\mathcal{K}$ is well-known [CLM, CPS, CCPS May2. Use of $\mathcal{K}$ in cohomology can also produce elegant results. In $[\mathrm{PPW}$ we use $\mathcal{K}$ to describe bases for certain cyclic unstable $\mathcal{A}$-modules. In particular, we show that the module $\mathcal{M}(n)$ which is the quotient of the free unstable $\mathcal{A}$-module on a generator $x_{2^{n}}$ of degree $2^{n}$ by the action of $\mathcal{A}\left(S q^{1}, \ldots, S q^{2^{n-2}}\right)$ can be expressed as a $\mathcal{K}$-module with basis the set $\left\{D_{I} x_{2^{n}}\right\}$ where $D_{I}$ is admissible and $I$ consists only of entries of the form 0 and powers of 2 that are less than $2^{n}$. We use this description to give the following global characterization of the Dickson algebras: The Dickson algebra $\mathcal{K}_{n+1, *}^{*}$ is the $\mathcal{K}$-algebra quotient of the free unstable $\mathcal{K}$-algebra on $\mathcal{M}(n)$ by the single additional relation $D_{2^{n-1}} D_{2^{n-1}} x_{2^{n}}=x_{2^{n}} D_{2^{n-1}} x_{2^{n}}$.

Finally, we close with the following particularly elegant formulation of the theorem of J. Lin [Li] about the action of the Steenrod algebra in the cohomology of finite $H$-spaces.

Let $Q H^{-i} X$ denote the indecomposable quotient of $H^{*} X$ in degree $-i(i \geq 0)$. Let $N_{j}$ be the set of those natural numbers with $j$ ones in their dyadic expansion, and let $Q H^{(j)} X=\bigoplus_{i \in N_{j}} Q H^{-i} X$. Clearly, $Q \widetilde{H}^{*} X=\bigoplus_{j} Q H^{(j)} X$. Then

Theorem 9.1. Let $X$ be a simply connected finite $H$-space, and let

$$
K=\left\{D_{1}^{i_{1}} D_{3}^{i_{2}} \ldots D_{2^{k}-1}^{i_{k}} \cdots \mid\left\{i_{k}\right\} \quad \text { is eventually zero }\right\}
$$


Then for each $j$,

$$
Q H^{(j)} X=K \cdot Q H^{-\left(2^{j}-1\right)} X
$$

The theorem may be proved by a straightforward induction from Lin's theorem, which says that under the hypotheses on $X$, any indecomposable in degree $-\left(2^{r+1} k+2^{r}-1\right)$ for $k>0$ is in the image of $S q^{2^{r} k}$ (which turns out to be just $\left.D_{2^{r}-1}\right)$.

But the theorem is perhaps better understood as follows, and perhaps even stated a little more strongly. Elements of $K$ acting on $x \in Q H^{-\left(2^{j}-1\right)} X$ retain exactly $j$ dyadic ones in the resulting degree (indecomposability is not needed for this), and moreover, every indecomposable $y \in Q H^{(j)} X$ arises in this way. In actual fact, for $y$ in fixed degree, the element $D_{I} \in K$, for which $y=D_{I} x$ is uniquely determined (provided we do not redundantly insert $D_{2^{j}-1}$, for which $D_{2^{j}-1} x=x$ ), and is easily calculated from the dyadic expansion.

For instance, any indecomposable $y$ in dyadic degree -101111100111 is the image of precisely $D_{2^{3}-1}^{2} D_{2^{8}-1}$ on an indecomposable $x$ in dyadic degree -111111111 . As the class $y$ is traced back towards $x$ by successive individual operations and intermediate classes, the number $l$ of trailing ones in each intermediate dyadic degree dictates the subscript $2^{l}-1$ of the next operation; and each operation always simply excises the rightmost zero in the expansion. Thus the lengths of blocks of consecutive zeros in the expansion of the degree of $y$ are the exponents, in reverse order, of $D_{I}$.

\section{REFERENCES}

[Ada] J.F. Adams, Infinite loop spaces, Princeton Univ. Press, 1978. MR 80d:55001

[Ade] J. Adem, The relations on Steenrod powers of cohomology classes, in Algebraic Geometry and Topology (ed. R.H. Fox), Princeton Univ. Press, 1957, pp. 191-238. MR 19:50c

[AK] S. Araki, T. Kudo, Topology of $H_{n}$-spaces and $H$-squaring operations, Mem. Fac. Sci. Kyusyu Univ. Ser. A 10 (1956), 85-120. MR 19:442b

[BJ] T.P. Bisson, A. Joyal, Q-rings and the homology of the symmetric groups, Contemporary Mathematics 202 (1997), 235-286. MR 98e:55021]

[BPW] T.P. Bisson, D.J. Pengelley, F. Williams, Stabilizing the lower operations for mod two cohomology, in Homotopy Invariant Algebraic Structures: A Conference in Honor of J. Michael Boardman (ed. J. P. Meyer, J. Morava, and W. J. Wilson), Contemporary Mathematics, to appear.

[BM] S.R. Bullett, I.G. Macdonald, On the Adem relations, Topology 21 (1982), 329-332. MR 83h:55035

[CCPS] H.E.A. Campbell, F.R. Cohen, F.P. Peterson, P.S. Selick, Self-maps of loop spaces. II, Trans. Amer. Math. Soc. 293 (1986), 41-51. MR 87e:55010b

[CPS] H.E.A. Campbell, F.P. Peterson, P.S. Selick, Self-maps of loop spaces. I, Trans. Amer. Math. Soc. 293 (1986), 1-39. MR 87e:55010a

[CLM] F.R. Cohen, T.J. Lada, J. P. May, The homology of iterated loop spaces, Lecture Notes in Mathematics 533 (1976), Springer, New York. MR 55:9096

[DL] E. Dyer, R.K. Lashof, Homology of iterated loop spaces, Amer. J. Math. 84 (1962), 35-88. MR 25:4523

[GP] V. Giambalvo, F.P. Peterson, A-generators for ideals in the Dickson algebra, to appear.

[GPR] V. Giambalvo, D.J. Pengelley, D.C. Ravenel, A fractal-like algebraic splitting of the classifying space for vector bundles, Trans. Amer. Math. Soc. 307 (1986), 433-455. MR 89c:55014

[HP] N.H.V. Hung, F.P. Peterson, A-generators for the Dickson algebra, Trans. Amer. Math. Soc. 347 (1995), 4687-4728. MR 96c:55022

[H] T. Hunter, On the homology spectral sequence for topological Hochschild homology, Trans. Amer. Math. Soc. 348 (1996), 3941-3953. MR 96m:55032 
[KL1] D. Kraines, T. Lada, The cohomology of the Dyer-Lashof algebra, in Proc., Northwestern Homotopy Theory Conference (Evanston, Ill., 1982), Contemporary Math. 19 (1983), Amer. Math. Soc., 145-152. MR 85b:55032

[KL2] D. Kraines, T. Lada, Applications of the Miller spectral sequence, in Proc. Conf. Current Trends in Algebraic Topology (1981), Canadian Math. Soc. Conf. Proc. 2, Part 1, 479-497, American Mathematical Society, 1982. MR 84f:55006

[Li] J.P. Lin, Steenrod connections and connectivity in $H$-spaces, Memoirs of the Amer. Math. Soc. 369 (1987), 1-87. MR 88m:55013

[Lo] L. Lomonaco, A phenomenon of reciprocity in the universal Steenrod algebra, Trans. Amer. Math. Soc. 330 (1992), 813-821. MR 92f:55027

[Mad] I. Madsen, On the action of the Dyer-Lashof algebra in $H_{*}(G)$, Pacific J. Math. 60 (1975), 235-275. MR 52:9228

[MM] I. Madsen, R. J. Milgram, The classifying spaces for surgery and cobordism of manifolds, Princeton Univ. Press, 1979. MR 81b:57014

[May1] J.P. May, A general algebraic approach to Steenrod operations, in Proc. Conf., The Steenrod Algebra and its Applications (ed. F. Peterson), Lecture Notes in Mathematics 168 (1970), Springer, New York, pp. 153-231. MR 43:6915

[May2] J.P. May, Homology operations on infinite loop spaces, in Proc. Conf., Algebraic Topology (Madison, 1970), Proc. Symp. Pure Math. 22, Amer. Math. Soc., 1971, pp. 171-185. MR 47:7740

[Mill] H. Miller, A spectral sequence for the homology of an infinite delooping, Pacific J. Math. 79 (1978), 139-155. MR 80g:55019

[Miln] J. Milnor, The Steenrod algebra and its dual, Annals of Math. (2) 67 (1958), 150-171. MR 20:6092

[Mu] H. Mui, Modular invariant theory and cohomology algebras of symmetric groups, Jour. Fac. Sci. Univ. Tokyo, Sect. 1A, 22 (1975), 319-369. MR 54:10440

[N] G. Nishida, Cohomology operations in infinite loop spaces, Proc. Japan Acad. 44 (1968), 104-109. MR 39:2156

[PPW] D.J. Pengelley, F. P. Peterson, F. Williams, A global structure theorem for the mod two Dickson algebras, and unstable cyclic modules over the Steenrod and Kudo-Araki-May algebras, to appear.

[PW1] D.J. Pengelley, F. Williams, Limits of algebras with shifting and a relationship between the Steenrod and Dyer-Lashof algebras, to appear.

[Pr] S.B. Priddy, Koszul Resolutions, Trans. Amer. Math. Soc. 152 (1970), 39-60. MR 42:346

[Po] M.M. Postnikov, The lower Steenrod algebra, Uspeckhi Mat. Nauk 49 (1994), 187-188. MR 95f:55016

[Sm] V.A. Smirnov, Radical Hopf algebras and the Steenrod algebra, Uspekhi Mat. Nauk 42 (1987), 245-246. MR 88k:55017

[Stee1] N.E. Steenrod, Homology groups of symmetric groups and reduced power operations, Proc. Nat. Acad. Sci. U.S.A. 39 (1953), 213-217. MR 14:1005d

[Stee2] N.E. Steenrod, Cohomology operations derived from the symmetric group, Comment. Math. Helv. 31 (1957), 195-218. MR 19:1069i

[Stee3] N.E. Steenrod, D.B.A. Epstein, Cohomology Operations, Princeton Univ. Press, 1962. MR 26:3056

[Stei] R. Steiner, Homology operations and power series, Glasgow Math. J. 24 (1983), 161-168. Corrigendum, Glasgow Math. J. 26 (1985), 105. MR 85e:55037, MR 86d:55023

[W] C. Wilkerson, A primer on the Dickson invariants, in Proc., Northwestern Homotopy Theory Conference (Evanston, Ill., 1982), Contemporary Math. 19 (1983), Amer. Math. Soc., 421-434. MR 85c:55017

Department of Mathematics, New Mexico State University, Las Cruces, New Mexico 88003

E-mail address: davidp@nmsu.edu

E-mail address: frank@nmsu.edu 